\title{
Time Scales of Submesoscale Flow Inferred from a Mooring Array
}

\author{
JÖRN CALLIES \\ California Institute of Technology, Pasadena, California \\ ROY BARKAN \\ Tel Aviv University, Tel Aviv, Israel, and University of California, Los Angeles, Los Angeles, California
}

Alberto Naveira Garabato

University of Southampton, Southampton, United Kingdom

(Manuscript received 24 October 2019, in final form 19 January 2020)

\begin{abstract}
While the distribution of kinetic energy across spatial scales in the submesoscale range $(1-100 \mathrm{~km})$ has been estimated from observations, the associated time scales are largely unconstrained. These time scales can provide important insight into the dynamics of submesoscale turbulence because they help quantify to what degree the flow is subinertial and thus constrained by Earth's rotation. Here a mooring array is used to estimate these time scales in the northeast Atlantic. Frequency-resolved structure functions indicate that energetic wintertime submesoscale turbulence at spatial scales around $10 \mathrm{~km}$ evolves on time scales of about 1 day. While these time scales are comparable to the inertial period, the observed flow also displays characteristics of subinertial flow that is geostrophically balanced to leading order. An approximate Helmholtz decomposition shows the order 10-km flow to be dominated by its rotational component, and the root-meansquare Rossby number at these scales is estimated to be 0.3 . This rotational dominance and Rossby numbers below one persist down to $2.6 \mathrm{~km}$, the smallest spatial scale accessible by the mooring array, despite substantially superinertial Eulerian evolution. This indicates that the Lagrangian evolution of submesoscale turbulence is slower than the Eulerian time scale estimated from the moorings. The observations therefore suggest that, on average, submesoscale turbulence largely follows subinertial dynamics in the 1-100-km range, even if Doppler shifting produces superinertial Eulerian evolution. Ageostrophic motions become increasingly important for the evolution of submesoscale turbulence as the scale is reduced-the root-mean-square Rossby number reaches 0.5 at a spatial scale of $2.6 \mathrm{~km}$.
\end{abstract}

\section{Motivation}

Recent observations of submesoscale flows have improved our understanding of their dynamics. The submesoscales, here referring to the spatial scales of about $1-100-\mathrm{km}$ wavelength, encompass the dynamical transition from energetic mesoscale eddies at a few hundred kilometers to high-frequency internal waves and mixed layer turbulence at scales smaller than $1 \mathrm{~km}$. The observations show submesoscale energy levels undergo a large seasonal cycle in the mixed layer and drop off rapidly with depth below the base of the mixed layer. Together with theoretical expectations, idealized models, and realistic numerical simulations, this suggests that submesoscale

Corresponding author: Jörn Callies, jcallies@caltech.edu turbulence is energized primarily by mixed layer baroclinic instabilities (Boccaletti et al. 2007; Fox-Kemper et al. 2008; Mensa et al. 2013; Sasaki et al. 2014; Callies et al. 2015, 2016; Buckingham et al. 2016; Thompson et al. 2016). Wintertime atmospheric forcing generates deep well-mixed surface layers, which in the presence of lateral buoyancy gradients provide a large reservoir of potential energy available for release. Subsequent to the conversion to submesoscale kinetic energy by the instability, turbulent scale interactions redistribute the energy across the submesoscale range and thus energize the full range of scales. Outside of winter and well below the mixed layer, this energization is absent and submesoscale turbulence is much weaker.

These dynamics have been inferred largely from spatial statistics of submesoscale flows: wavenumber 
spectra estimated at different depths and seasons from shipboard current profilers and towed instruments (Wang et al. 2010; Callies and Ferrari 2013; Shcherbina et al. 2013; Callies et al. 2015; Rocha et al. 2016; Chereskin et al. 2019). These spatial statistics, however, provide only indirect and at best qualitative information on the time scales of the flow. Helmholtz and wavevortex decompositions of the observed wavenumber spectra allow a scale-by-scale categorization of the observed flow into whether it is dominated by slow geostrophic flow or fast internal waves (Bühler et al. 2014). While this categorization provides crucial clues for the dynamical interpretation of the observations, it offers no information on the time scales of the balanced submesoscale turbulence that is energized in winter.

Access to the time scales of submesoscale flows would allow important insight into their dynamics. The assumption that submesoscale turbulence evolves on sufficiently long time scales to be to leading order geostrophically balanced, that is, that the Rossby number is sufficiently small, has guided the interpretation of the observations in terms of geostrophic turbulence theory (e.g., Le Traon et al. 2008; Callies et al. 2015, 2016), and it is a core assumption of the wave-vortex decomposition that has been employed to aid this interpretation (Bühler et al. 2014). Understanding at what scale this assumption breaks down is important because this is where the dynamical constraint imposed by Earth's rotation is at least partially overcome (e.g., Thomas et al. 2008; McWilliams 2016). At this scale, the horizontal divergence assumes the same order of magnitude as the vertical vorticity, such that vertical velocities reach the magnitudes expected from an aspect ratio scaling-instead of being reduced by another small factor equal to the Rossby number, as is the case in quasigeostrophic dynamics (e.g., Pedlosky 1987; Barkan et al. 2019). It has been argued that the resulting vertical flow crucially enhances the vertical exchange between the surface and interior ocean (e.g., Ferrari 2011; Omand et al. 2015; Mahadevan 2016) and that the associated horizontal convergence fundamentally changes how buoyant particles and tracers are dispersed (D'Asaro et al. 2018). The spatial scale at which the Rossby number reaches order one is also where kinetic energy is expected to experience a substantial forward cascade, instead of being trapped at large scales as is the case in geostrophic turbulence (e.g., Müller et al. 2005; Capet et al. 2008; Molemaker et al. 2010).

To address these questions, Callies et al. (2015) estimated from observations of submesoscale turbulence a scale-dependent Rossby number $\operatorname{Ro}(k)=[f \tau(k)]^{-1}$, which compares the inertial time scale $f^{-1}$ to the advective time scale $\tau(k)=\left[k^{3}\left\langle|\hat{\mathbf{u}}(k)|^{2}\right\rangle / 2\right]^{-1 / 2}$. Here $k$ is the wavenumber measured along a ship track, and $\left\langle|\hat{\mathbf{u}}(k)|^{2}\right\rangle / 2$ is the kinetic energy spectrum. In the western subtropical North Atlantic, where wintertime submesoscale turbulence is particularly energetic, this Rossby number increases with $k^{1 / 2}$ from $\operatorname{Ro}(k) \approx 0.1$ at the wavelength $\lambda=2 \pi / k=100 \mathrm{~km}$ to $\operatorname{Ro}(k) \approx 1$ at $\lambda=1 \mathrm{~km}$, suggesting that small-Rossby-number dynamics break down qualitatively at a wavelength of order $1 \mathrm{~km}$. Consistent with this result, Shcherbina et al. (2013) found the vertical vorticity measured at scales of order $1 \mathrm{~km}$ to have magnitudes of the same order as $f$ and to be significantly skewed to positive values.

In apparent contradiction with these observations, however, a recent submesoscale-permitting numerical simulation of the global ocean showed submesoscale turbulence to have time scales much shorter than the inertial period (Rocha et al. 2016; Qiu et al. 2018; Torres et al. 2018). In wavenumber-frequency spectra of kinetic energy, which offer a comprehensive and concise description of the spatial and temporal scales of submesoscale flows, submesoscale turbulence was found to have energy concentrated along a straight line in logarithmic wavenumber-frequency space (Torres et al. 2018; cf. Fig. 5 here). In the Kuroshio extension region, which has submesoscale turbulence of similar characteristics and energy levels as in the western subtropical North Atlantic the observations of Shcherbina et al. (2013) and Callies et al. (2015) were from, this line crosses the inertial frequency at a wavelength of about $50 \mathrm{~km}$. Flow at spatial scales smaller than $50 \mathrm{~km}$ therefore evolves on time scales that are superinertial. In contrast to the observational Rossby number estimates, these wavenumber-frequency spectra inferred from the simulation thus seem to suggest that describing submesoscale turbulence with subinertial dynamics is hopeless.

This apparent contradiction between the observations suggesting low-Rossby-number dynamics at scales larger than order $1 \mathrm{~km}$ and the simulation suggesting superinertial evolution across most of the submesoscale range indicates a fundamental lack of understanding of the time evolution of submesoscale turbulence. There are at least two possible resolutions. First, it remains unclear whether observations support the inference that submesoscale turbulence evolves on time scales comparable to and shorter than the inertial period. While the simulated mesoscale and submesoscale turbulence has been shown to have energy levels comparable to observations (Rocha et al. 2016; Qiu et al. 2018; Erickson et al. 2020), there are so far no observational constraints on the time scales of this turbulence. Second, it remains unclear whether the rapid Eulerian evolution diagnosed in wavenumber-frequency spectra is indeed inconsistent 


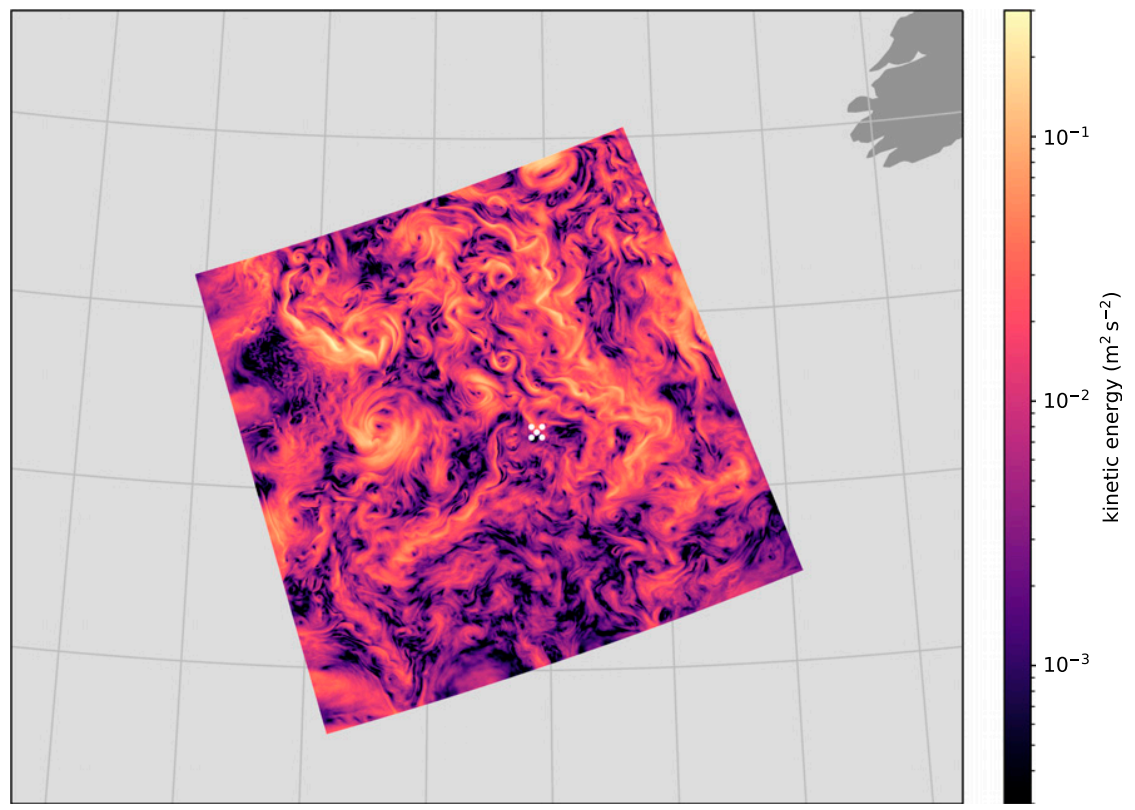

FIG. 1. Location of the OSMOSIS array in the northeast Atlantic. The shading shows a surface kinetic energy snapshot from the numerical simulation, and the white dots mark the locations of the central and outer moorings. The landmass in the northeast corner of the map is Ireland.

with low-Rossby-number dynamics. Slowly evolving submesoscale flow could be swept past an Eulerian observer and thus appear at an Eulerian period that is much shorter than the time scale of Lagrangian evolution.

In this paper, we present indirect support for this second interpretation by inferring time scales of submesoscale turbulence from observations and confirming the rapid Eulerian evolution. Ideally, one would seek an observational estimate of the wavenumber-frequency spectrum, but currently no observational estimate that extends into the submesoscale range is available for the open ocean. ${ }^{1}$ The logistical demands for observing such a spectrum are substantial: if standard spectral techniques were to be used, estimating the spectrum across the relevant time and space scales would require simultaneous hourly observations in at least 200 locations along a line, with a spacing of $0.5 \mathrm{~km}$ and sustained for at least several months.

While a full wavenumber-frequency spectrum is currently not available, partial information on the spatial and temporal scales of submesoscale turbulence can be obtained from the Ocean Surface Mixing, Ocean Submesoscale Interaction Study (OSMOSIS) mooring array that was deployed in the northeast Atlantic from September 2012 to September 2013 (Buckingham et al. 2016; Yu et al. 2019). We here

\footnotetext{
${ }^{1}$ For a mesoscale wavenumber-frequency spectrum obtained from altimetry, see Wunsch and Stammer (1995) and Wunsch (2009).
}

calculate frequency-resolved structure functions from velocity differences across mooring pairs. In addition to the usual temporal information, these statistics provide spatial information at scales corresponding to the separation distance between the moorings. The OSMOSIS array consisted of nine moorings that were arranged in two squares centered on a central mooring (cf. Figs. 1 and 3). The outer square had a side length of about $13 \mathrm{~km}$; the inner square had a side length of about $2 \mathrm{~km}$. With this arrangement, mooring pairs separated by about $1-20 \mathrm{~km}$ are available, which allows us to make inferences about spatial scales corresponding to wavelengths of about $2-40 \mathrm{~km}$.

The paper is organized as follows. Section 2 gives an overview of the OSMOSIS observations. Section 3 discusses frequency-resolved structure functions, the key quantity to be diagnosed from observations, and describes how they are related to wavenumber-frequency spectra. Section 4 introduces an approximate Helmholtz decomposition that can be applied to the observed frequency-resolved structure functions to aid the dynamical interpretation. Section 5 describes the results obtained from the mooring array and offers a dynamical interpretation. Section 6 summarizes the dynamical insight gleaned from the observations.

\section{Observations}

Nine subsurface moorings were deployed as part of the OSMOSIS project (Buckingham et al. 2016; Yu et al. 2019). 
TABLE 1. Nominal depths (m) of the acoustic current meters.

\begin{tabular}{lcc}
\hline & Shallow & Deep \\
\hline Central & 51 & 528 \\
NE inner & 56 & 531 \\
NW inner & 28 & 494 \\
SW inner & 29 & 497 \\
SE inner & 41 & 510 \\
NE outer & 62 & \\
NW outer & 59 & 535 \\
SW outer & 43 & 522 \\
SE outer & 53 & 526 \\
\hline
\end{tabular}

They were each equipped with a number of Nortek Aquadopp acoustic current meters distributed across the upper ocean. We restrict our analysis to the current meters located closest to 50- and 500-m depth. The nominal depths of the selected instruments are listed in Table 1. Despite the differences in nominal depth, we treat these instruments as if they were at the same depth and subsequently refer to them as the $50-$ and $500-\mathrm{m}$ instruments. The instrument closest to $500 \mathrm{~m}$ failed on the northeast (NE) outer mooring, so we only have eight instruments at that depth. The moorings occasionally experienced substantial knockdown by the currents, but we estimate this has a negligible effect on the statistics presented below (appendix).

The current meter data have a time resolution of $10 \mathrm{~min}$. We linearly interpolate the data onto a common time grid with the same 10-min spacing. We separate the data into four time segments that roughly correspond to the fall, winter, spring, and summer seasons (Fig. 2). The fall season starts on 18 September 2012, and all seasons are 140 days long. We overlap the seasons by $50 \%$ because the spectral analysis used below requires windowing; the overlap allows us to take full advantage of the information content of the data.

The current meters at 50-m depth are in the seasonal thermocline in summer and in the mixed layer in winter, whereas the current meters at 500-m depth are at the lower edge of the subpolar mode water layer yearround (Fig. 2c). This is revealed by hydrographic data collected by gliders that were deployed in the region over the full period covered by the mooring measurements (Thompson et al. 2016). The sharp seasonal thermocline deepens from about $50 \mathrm{~m}$ at the beginning of the time series in September 2012 to $100 \mathrm{~m}$ in November. The seasonal thermocline subsequently disappears and leaves the subpolar mode water layer exposed to the atmosphere. This weakly stratified layer reaches down to the permanent thermocline below about $600-\mathrm{m}$ depth. A new seasonal thermocline starts forming in the spring of 2013, first intermittently in May, then permanently in June.
The frequency-resolved structure functions introduced below are calculated from mooring pairs and averaged over a collection of pairs of similar lateral separation. We group the 36 mooring pairs into nine collections with similar separation using $k$-means clustering (Fig. 3). The collections have average separations ranging from $18.7 \mathrm{~km}$, corresponding to the diagonals of the outer array, to $1.3 \mathrm{~km}$, corresponding to the pairs formed between the eastern inner moorings and the central mooring.

\section{Frequency-resolved structure functions}

At every available mooring location $\mathbf{x}$, we can calculate the temporal Fourier transform of the measured velocity component $u$ :

$$
\tilde{u}(\mathbf{x}, \omega)=\int_{-\infty}^{\infty} u(\mathbf{x}, t) e^{-i \omega t} d t .
$$

For simplicity, we here use the notation for an infinitely long and continuously sampled signal $u$; in practice, the continuous Fourier transform is replaced by a discrete Fourier transform, and the finite time series is multiplied by a Hann window to minimize spectral leakage. As usual, we can estimate the frequency spectrum $\left\langle|\tilde{u}(\omega)|^{2}\right\rangle$ from (1), where the angle brackets denote an expectation value that in practice corresponds to an average over the different moorings. We assume isotropy and homogeneity, by which the frequency spectrum becomes independent of location and the orientation of the coordinate system.

Having access to the temporal Fourier transforms at mooring pairs located at $\mathbf{x}$ and $\mathbf{x}+\mathbf{r}$ (Fig. 4a), we can also calculate the frequency-resolved structure function

$$
\Delta^{u}(r, \omega) \equiv \frac{1}{2}\left\langle|\tilde{u}(\mathbf{x}+\mathbf{r}, \omega)-\tilde{u}(\mathbf{x}, \omega)|^{2}\right\rangle .
$$

The angle brackets again denote an expectation value, now corresponding in practice to an average over mooring pairs that are separated by similar distances, that is, that are in the same collection of pairs (Fig. 3).

By the homogeneity and isotropy assumptions, the frequency-resolved structure function (2) only depends on the separation $r=|\mathbf{r}|$ and the orientation of the velocity component relative to $\mathbf{r}$. We define $u=\mathbf{u} \cdot \mathbf{r} / r$ as the longitudinal component and $v=\mathbf{u} \cdot(\mathbf{z} \times \mathbf{r}) / r$ as the transverse component relative to the respective mooring pair, where $\mathbf{z}$ is the vertical unit vector (Fig. 4a). The transverse frequency-resolved structure function $\Delta^{v}(r, \omega)$ is defined analogously to (2). We take advantage of the difference between the longitudinal and 

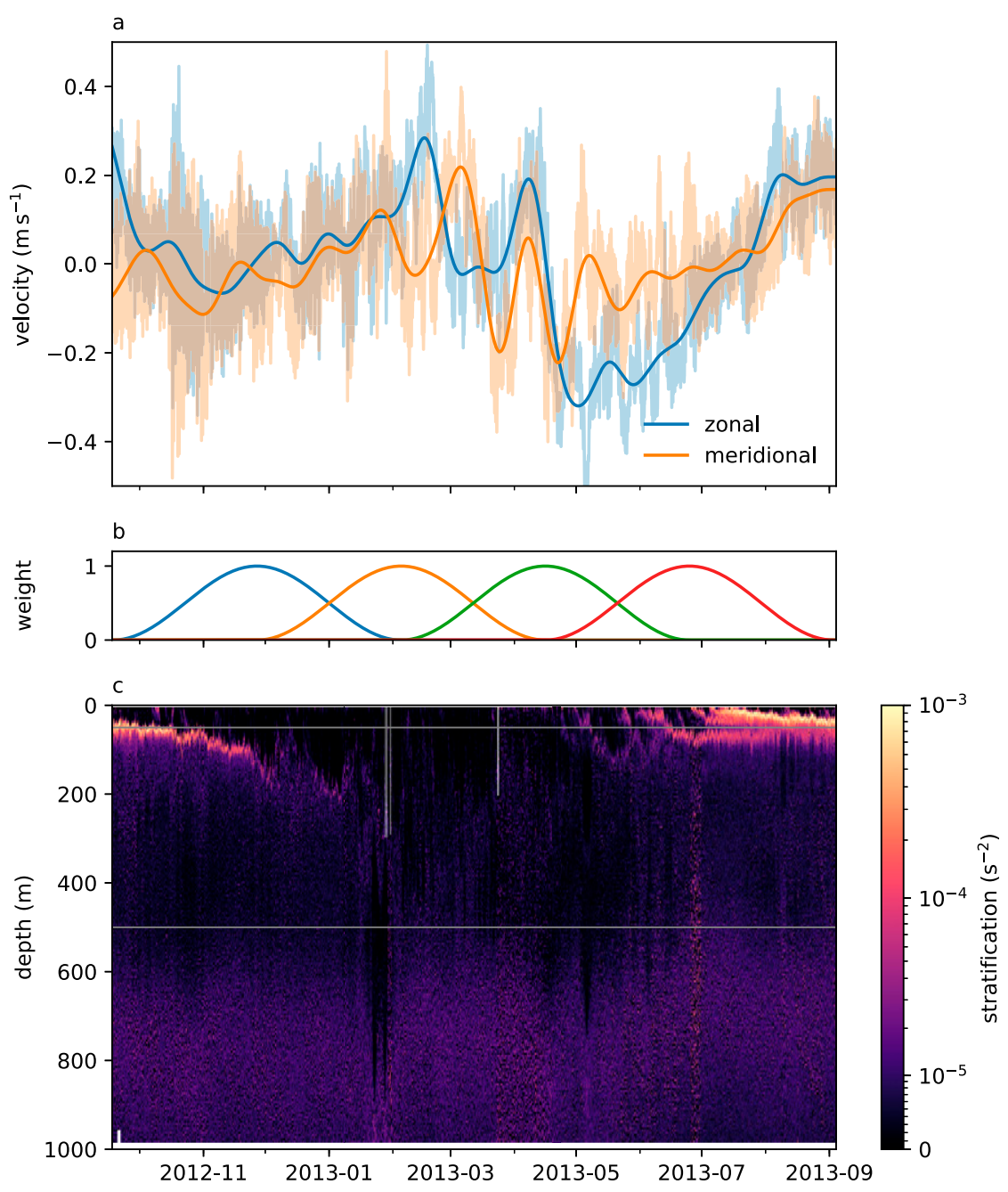

FIG. 2. OSMOSIS observations of a full seasonal cycle. (a) Velocities from the central mooring at 50-m depth (raw: transparent; filtered to 20 days: solid). (b) Hann windows for the four seasons (fall, winter, spring, summer). (c) Stratification inferred from gliders, showing the evolution of the seasonal thermocline in the top $200 \mathrm{~m}$ and the permanent thermocline below $600 \mathrm{~m}$. Note the arsinh-scaled color map. The horizontal lines show the depths of the current meters analyzed in this study.

transverse statistics in the approximate Helmholtz decomposition introduced in section 4 .

The frequency-resolved structure function encodes spatial as well as temporal information. It is related to the wavenumber-frequency spectrum $\left\langle|\hat{u}(\kappa, \omega)|^{2}\right\rangle$ through

$$
\begin{aligned}
\Delta^{u}(r, \omega) & =\left\langle|\tilde{u}(\omega)|^{2}\right\rangle-\left\langle\tilde{u}^{*}(\mathbf{x}, \omega) \tilde{u}(\mathbf{x}+\mathbf{r}, \omega)\right\rangle \\
& =\iint\left\langle|\hat{u}(\mathbf{k}, \omega)|^{2}\right\rangle\left(1-e^{i \mathbf{k} \cdot \mathbf{r}}\right) d^{2} \mathbf{k} \\
& =\int_{0}^{\infty}\left\langle|\hat{u}(\kappa, \omega)|^{2}\right\rangle F_{0}(\kappa r) d \kappa
\end{aligned}
$$

where the kernel in the last expression is

$$
F_{0}(\xi)=1-J_{0}(\xi),
$$

and $J_{0}$ denotes the Bessel function of the first kind and zeroth order. This identity follows by making use of the Wiener-Khinchin theorem, Bessel's integral, and the isotropy assumption (e.g., Yaglom 1962). We denote the two-dimensional wavenumber vector by $\mathbf{k}$ and its magnitude by $\kappa=|\mathbf{k}|$. We use a standard normalization for the two-dimensional isotropic spectrum:

$$
2 \pi \kappa\left\langle|\hat{u}(\mathbf{k}, \omega)|^{2}\right\rangle=\left\langle|\hat{u}(\kappa, \omega)|^{2}\right\rangle,
$$

which ensures that integrals over the respective wavenumber domains return the frequency spec$\operatorname{trum}\left\langle|\tilde{u}(\omega)|^{2}\right\rangle$. 


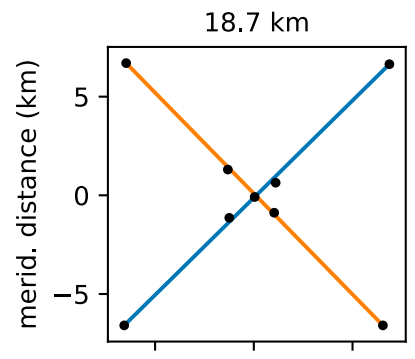

$9.4 \mathrm{~km}$

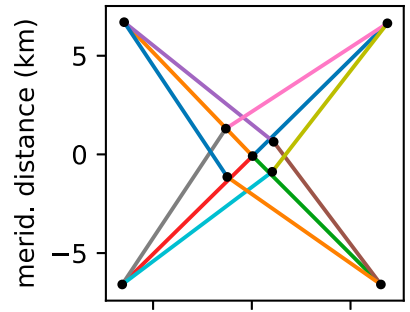

$2.4 \mathrm{~km}$

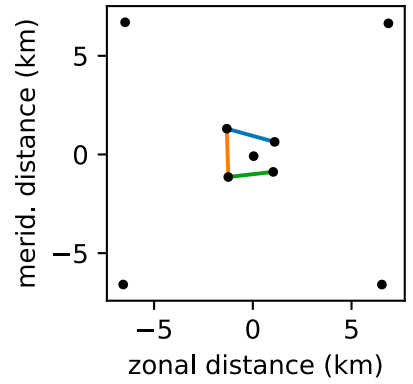

$13.2 \mathrm{~km}$

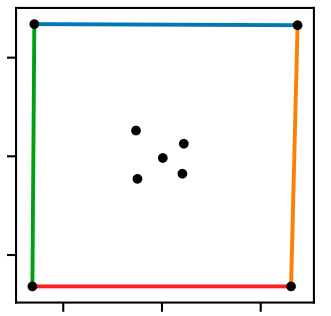

$7.8 \mathrm{~km}$

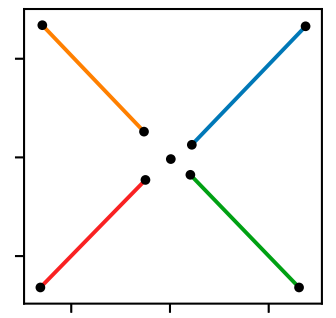

$1.7 \mathrm{~km}$

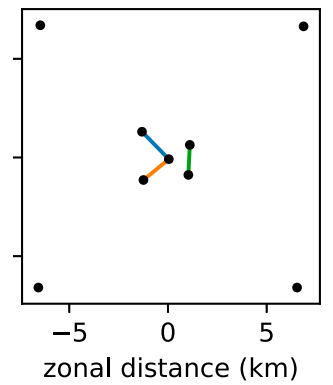

$10.9 \mathrm{~km}$

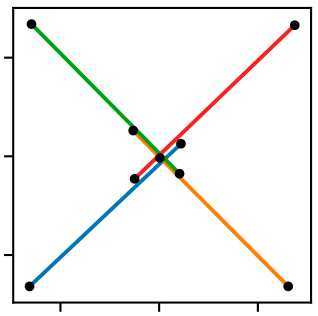

$3.1 \mathrm{~km}$

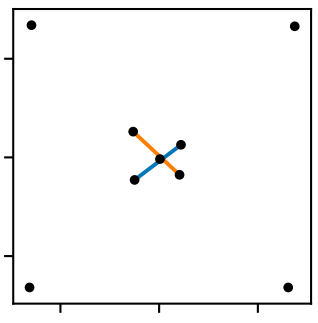

$1.3 \mathrm{~km}$

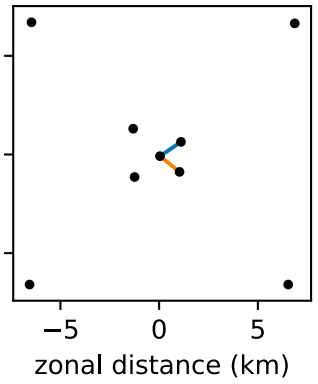

FIG. 3. Collections of mooring pairs with similar separations. Each subplot corresponds to a collection, with the mean separation $r$ indicated in the title. The pairs are marked with the colored lines.

Note that in principle the cross-spectrum $\left\langle\tilde{u}^{*}(\mathbf{x}, \omega) \tilde{u}(\mathbf{x}+\mathbf{r}, \omega)\right\rangle$ can be inverted for the wavenumber-frequency spectrum with a Hankel transform (cf. LaCasce 2016):

$$
\left\langle|\hat{u}(\kappa, \omega)|^{2}\right\rangle=\int_{0}^{\infty}\left\langle\tilde{u}^{*}(\mathbf{x}, \omega) \tilde{u}(\mathbf{x}+\mathbf{r}, \omega)\right\rangle J_{0}(\kappa r) \kappa r d r
$$

But performing this integration in separation space is difficult with the observations available to us. From the OSMOSIS array, the cross-spectrum can be estimated at only nine separation distances that are not optimally spaced for a Hankel transform. (Note above that the isotropy assumption assures that the cross-spectrum is real.)

The identity (3) will be a central tool in our analysis of the OSMOSIS observations: using it, we will gather information on the underlying wavenumber-frequency spectrum. The identity tells us that the frequency-resolved structure function at separation distance $r$ samples the wavenumber-frequency spectrum $\left\langle|\hat{u}(\kappa, \omega)|^{2}\right\rangle$ with the weighting kernel $F_{0}(\kappa r)$. The kernel is small for $\kappa r \ll 1$, rises to order unity around $\kappa r \sim \pi$ (where $r$ corresponds to the half-wavelength $\pi / \kappa$ ), and oscillates around unity with decreasing amplitude for larger $\kappa r$ (Fig. 5b). The frequency-resolved structure function at different separation distances $r$ thus contains partial information on the underlying wavenumberfrequency spectrum.

We illustrate this information content using a numerical simulation, from which we can calculate both the full wavenumber-frequency spectrum and the frequency-resolved structure functions (Fig. 5). The simulation setup is similar to the North Atlantic simulation described in Chelton et al. (2019); the detailed setup and further analysis will be described in a forthcoming publication-here we use this simulation for illustration only. We sample the model at the OSMOSIS mooring locations and 50-m depth, and we use the 140-day period corresponding to the winter season. All Fourier coefficients are calculated by multiplying the 140-day time segments by Hann windows and subsequently applying a discrete Fourier transform. The averages are applied 
a

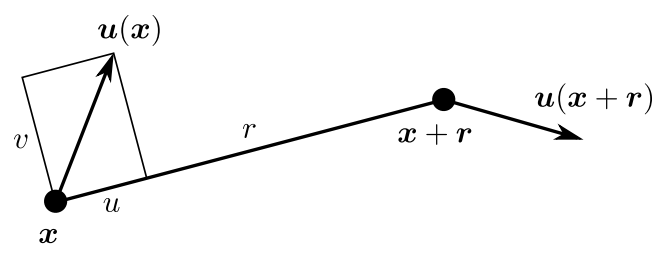

b

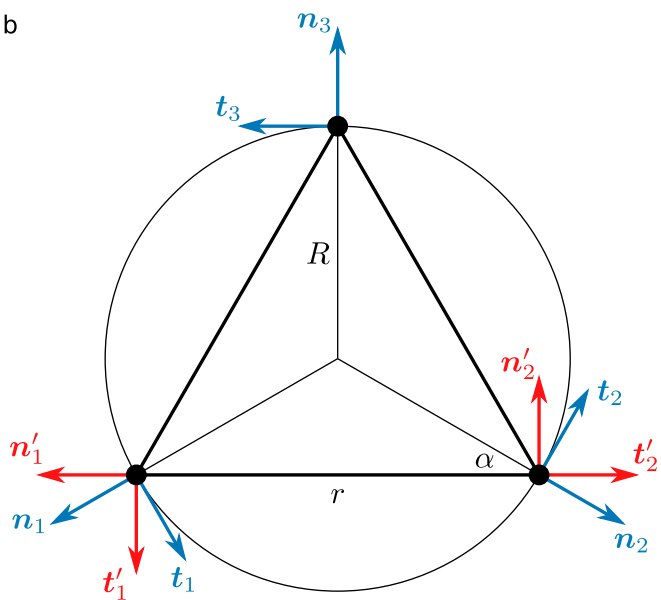

FIG. 4. Geometry of velocity measurements at mooring pairs and (hypothetical) mooring triplets. (a) Velocities u at moorings located at $\mathbf{x}$ and $\mathbf{x}+\mathbf{r}$. (b) Mooring triplet with side length $r$ and radius $R$. The tangent and normal vectors $\mathbf{t}$ and $\mathbf{n}$ with respect to the triangle are shown in blue, the ones with respect to the mooring pair are shown in red.

over all moorings for the frequency spectrum and over all mooring pairs in the respective pair collection for the frequency-resolved structure functions. Averaging in frequency bins is applied to reduce the estimation error. The normalization is such that $\left\langle|\hat{u}(\omega)|^{2}+|\hat{v}(\omega)|^{2}\right\rangle / 2=$ $\left[\Delta^{u}(\infty, \omega)+\Delta^{v}(\infty, \omega)\right] / 2$, assuming the flow is decorrelated between infinitely spaced moorings.

The wavenumber-frequency spectrum displays features familiar from the previous analyses of Rocha et al. (2016), Savage et al. (2017), and Torres et al. (2018) (Fig. 5a). Energy that is broadband in both wavenumber and frequency space falls along a slanted line in logarithmic wavenumber-frequency space, ranging from a few hundred kilometers to the model's dissipation scales and from time scales of months to superinertial frequencies. Energy at the inertial frequency is concentrated at large spatial scales. Energy at the semidiurnal tidal frequency is also enhanced at large scales. Energy at superinertial frequencies shows some enhancements along the dispersion curves of low-mode internal waves. A lot of this information can be extracted with frequency-resolved structure functions.
At the lowest frequencies, the flow is dominated by spatial scales (wavelengths) larger than $2 r=37.4 \mathrm{~km}$, the largest scale sampled by the mooring array. This means that the flow is correlated across the corresponding mooring pairs, and the frequency-resolved structure function at $r=18.7 \mathrm{~km}$ is reduced below the frequency spectrum (Fig. $5 \mathrm{c}$ ). In other words, the weighting kernel $F_{0}(\kappa r)$ corresponding to $r=18.7 \mathrm{~km}$ misses a large part of the energy at these lowest frequencies (Fig. 5b). At smaller separation, the flow is even more strongly correlated across mooring pairs, that is, the kernels miss even more of the energy, and the frequency-resolved structure functions are suppressed even more.

As the frequency increases, the dominant spatial scale decreases. Around $\omega=3 \times 10^{-6} \mathrm{cps}$, the energy is dominated by spatial scales comparable to the larger mooring separations of order $10 \mathrm{~km}$. This means that the flow becomes decorrelated between the respective moorings, and the weighting kernels start sampling the entire energy-the frequency-resolved structure functions converge to the frequency spectrum (coldcolored lines in Fig. 5c). At the same frequencies, the flow is still correlated across moorings separated by distances of order $1 \mathrm{~km}$ and the corresponding frequency-resolved structure functions are still suppressed (warm-colored lines in Fig. 5c).

At the inertial and tidal frequencies, the energy is again dominated by scales larger than those captured by the mooring array. The frequency-resolved structure functions capture this: at all separations, they are reduced compared to the frequency spectrum. At the inertial and semidiurnal tidal frequencies, the pairs separated by order $10 \mathrm{~km}$ still feature peaks, reflecting the extension of the inertial and tidal flow to these spatial scales. The pairs separated by order $1 \mathrm{~km}$ show less enhancement at these frequencies because there is less of an inertial or tidal signal left at the corresponding spatial scales.

At the highest frequencies, the energy starts being dominated by order $1 \mathrm{~km}$ scales, such that the flow becomes decorrelated across all mooring pairs. The frequency-resolved structure functions indicate this reduction in scale by the successive convergence toward the frequency spectrum.

Note that the design of the mooring array entails a gap in logarithmic separation space (Figs. 3 and 5b). The pairs formed by the outer moorings and the closest inner moorings have a much larger separation $(r=7.8 \mathrm{~km})$ than the pairs formed by the diagonals of the inner moorings $(r=3.1 \mathrm{~km})$. The gaps in the frequency-resolved structure functions between these separations are thus the result of the sampling pattern and have no physical significance (Fig. 5c). 

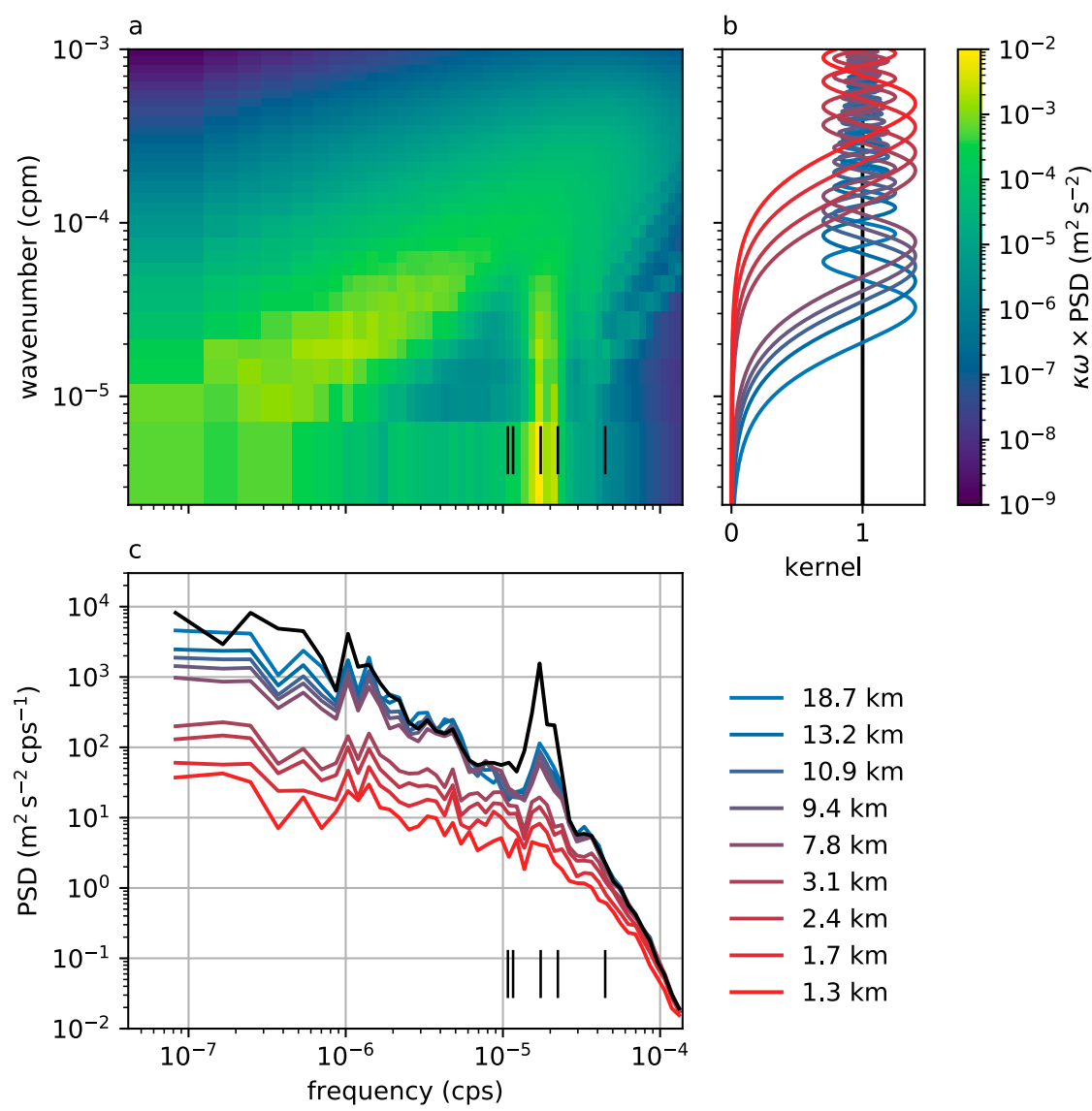

FIG. 5. Illustration of the information frequency-resolved structure functions provide about the underlying wavenumber-frequency spectrum. (a) The wavenumber-frequency spectrum estimated from a numerical model simulation. (b) The kernels $F_{0}(\kappa r)$ for the separations $r$ available from the OSMOSIS array (see legend). (c) The frequency-resolved structure functions for the same separations, estimated from a virtual mooring array. Also shown is the frequency spectrum, which is an integral over all wavenumbers with a unit weight. Short black vertical lines mark the two lunar diurnal frequencies, the inertial frequency, the lunar semidiurnal frequency, and the first harmonic of the lunar semidiurnal frequency.

\section{Single-separation Helmholtz decomposition}

An important discriminating property of submesoscale flow is the degree to which it is rotational or divergent. Given appropriate boundary conditions, there is a unique streamfunction $\psi$ and velocity potential $\phi$, such that the horizontal flow $\mathbf{u}$ can be decomposed as follows:

$$
\mathbf{u}=\mathbf{z} \times \nabla \psi+\nabla \phi .
$$

One can distinguish between the rotational and divergent components by exploiting that they produce different statistics for the longitudinal and transverse velocities. For a mooring pair, it is intuitive that divergent flow generates more variance of the longitudinal velocity difference and that rotational flow generates more variance of the transverse velocity difference. We exploit this property to estimate the rotational and divergent components.

Differences in the statistics of transverse and longitudinal velocity components were used by Bühler et al. (2014) to derive an exact Helmholtz decomposition calculable from one-dimensional wavenumber spectra. Lindborg (2015) reformulated that decomposition for structure functions and simplified the decomposition into the compact formulas

$$
\begin{aligned}
& \Delta_{\text {rot }}(r)=\Delta^{v}(r)+\int_{0}^{\infty}\left[\Delta^{v}(s)-\Delta^{u}(s)\right] \frac{d s}{s}, \\
& \Delta_{\text {div }}(r)=\Delta^{u}(r)+\int_{0}^{\infty}\left[\Delta^{u}(s)-\Delta^{v}(s)\right] \frac{d s}{s},
\end{aligned}
$$

where $\Delta_{\text {rot }}(r)$ and $\Delta_{\text {div }}(r)$ denote the structure functions of the rotational and divergent velocity components, 
respectively. We drop the frequency dependence in this section for notational compactness, but we will apply decompositions of this type to the frequency-resolved structure functions estimated from OSMOSIS data. We note in passing that the formulas derived below equally apply to purely spatial data, for example, those obtained from surface drifters (although additional complications arise from the biased sampling of drifters; Choi et al. 2017; Pearson et al. 2019).

It is difficult to apply the exact Helmholtz decomposition (8)-(9) to the mooring observations. For every separation distance $r$, these formulas require an integration over structure functions at all smaller separation distances $s \leq r$. One could approximate that integral for the larger mooring separations because a number of smaller separations are available, but the integration is entirely impossible for the smallest mooring separations.

Under certain conditions (to be made explicit below), however, one can obtain an approximate Helmholtz decomposition from knowledge of the transverse and longitudinal structure functions at a single separation distance $r$ only:

$$
\Delta_{\text {rot }}(r)=\frac{3}{2} \Delta^{v}(r)-\frac{1}{2} \Delta^{u}(r), \quad \Delta_{\text {div }}(r)=\frac{3}{2} \Delta^{u}(r)-\frac{1}{2} \Delta^{v}(r) .
$$

These formulas share with (8)-(9) the sensible property that the divergent and rotational components sum up to the full velocity structure function:

$$
\Delta_{\text {rot }}(r)+\Delta_{\text {div }}(r)=\Delta^{u}(r)+\Delta^{v}(r) .
$$

We detail three ways to derive the formulas in (10). In the first derivation, we assume that the flow is dominated by scales larger than $r$, which allows an approximation to (8) and (9). In the second derivation, we consider the divergence and vorticity calculated from an equilateral mooring triangle. In the third derivation, we consider the kernels through which the longitudinal and transverse structure functions are related to the rotational and divergent components of the kinetic energy spectrum, and we find the linear combination that isolates the respective part in the asymptotic limit $\kappa r \ll 1$. While these three derivations all lead to the same result, we find that describing all three builds intuition for the meaning and limitations of (10).

It is possible to find somewhat more accurate formulas than (10) if one takes advantage of the statistics at more than one separation distance. We here discuss the singleseparation decomposition (10) only because we find that its lack of accuracy is easily outweighed by its simplicity and interpretability.

\section{a. Large-scale limit of the exact decomposition}

If the signal is dominated by scales larger than the separation $r$, structure functions have an $r^{2}$ dependence (e.g., Babiano et al. 1985). Equation (8) then yields

$$
\begin{aligned}
\Delta_{\text {rot }}(r) & =\Delta^{v}(r)+\left[\Delta^{v}(r)-\Delta^{u}(r)\right] \int_{0}^{r}\left(\frac{s}{r}\right)^{2} \frac{d s}{s} \\
& =\frac{3}{2} \Delta^{v}(r)-\frac{1}{2} \Delta^{u}(r) .
\end{aligned}
$$

The analogous calculation for $\Delta_{\text {div }}(r)$ leads to the divergent component in (10). The single-separation decomposition (10) is thus exact in the limit of the flow being dominated by scales larger than the separation $r$.

If $\Delta^{u}(s)$ and $\Delta^{v}(s)$ followed a known power law $s^{m}$ for $0<s<r$ with $0<m<2$, (8) and (9) would instead yield the exact decomposition

$$
\begin{aligned}
& \Delta_{\mathrm{rot}}(r)=\frac{m+1}{m} \Delta^{v}(r)-\frac{1}{m} \Delta^{u}(r), \\
& \Delta_{\mathrm{rot}}(r)=\frac{m+1}{m} \Delta^{u}(r)-\frac{1}{m} \Delta^{v}(r) .
\end{aligned}
$$

We here exclusively use (10) because it can be applied if the structure functions are known at a single separation only. The error incurred by assuming the nonlocal limit is further discussed below.

\section{b. Triangular mooring array}

The same formulas arise from calculating the structure function of the rotational and divergent components of the flow estimated form a triplet of moorings arranged in an equilateral triangle with side length $r$ (Fig. 4). To illustrate this, we consider diagnosing the rotational part of the flow. The magnitude of this rotational component is estimated from the moorings as the flow along the circle circumscribing the triangle:

$$
u_{\mathrm{rot}}=\frac{1}{\sqrt{3}} \sum_{i=1}^{3} \mathbf{u}_{i} \cdot \mathbf{t}_{i}
$$

where $\mathbf{x}_{i}$ and $\mathbf{t}_{i}$ are the position vector and tangent unit vector of mooring $i$ (tangent to the circumcircle of the triangle, pointing in the counterclockwise direction; see Fig. $4 \mathrm{~b})$, and $\mathbf{u}_{i}=\mathbf{u}\left(\mathbf{x}_{i}\right)$ is the velocity vector at mooring $i$. The normalization factor is chosen such that

$$
\left\langle u_{\text {rot }}^{2}\right\rangle+\left\langle u_{\text {div }}^{2}\right\rangle=\Delta^{u}(r)+\Delta^{v}(r),
$$

with a similarly defined $u_{\text {div }}$ [cf. (11)]. The variance of the such-defined rotational component of the flow 
matches the formula for the rotational component in the single-separation Helmholtz decomposition:

$$
\begin{aligned}
\left\langle u_{\mathrm{rot}}^{2}\right\rangle= & \frac{1}{2}\left\langle|\mathbf{u}|^{2}\right\rangle+\frac{2}{3} \sum_{i=1}^{3} \sum_{j=i+1}^{3}\left\langle\left(\mathbf{u}_{i} \cdot \mathbf{t}_{i}\right)\left(\mathbf{u}_{j} \cdot \mathbf{t}_{j}\right)\right\rangle \\
= & \frac{1}{2}\left\langle|\mathbf{u}|^{2}\right\rangle+2\left\langle\left(\mathbf{u}_{1} \cdot \mathbf{t}_{1}\right)\left(\mathbf{u}_{2} \cdot \mathbf{t}_{2}\right)\right\rangle \\
= & \frac{1}{2}\left\langle|\mathbf{u}|^{2}\right\rangle+2 \cos ^{2} \alpha\left\langle\left(\mathbf{u}_{1} \cdot \mathbf{t}_{1}^{\prime}\right)\left(\mathbf{u}_{2} \cdot \mathbf{t}_{2}^{\prime}\right)\right\rangle \\
& -2 \sin ^{2} \alpha\left\langle\left(\mathbf{u}_{1} \cdot \mathbf{n}_{1}^{\prime}\right)\left(\mathbf{u}_{2} \cdot \mathbf{n}_{2}^{\prime}\right)\right\rangle \\
= & \frac{3}{2} \Delta^{v}(r)-\frac{1}{2} \Delta^{u}(r),
\end{aligned}
$$

so setting $\Delta_{\text {rot }}(r)=\left\langle u_{\text {rot }}^{2}\right\rangle$ recovers (10). In this calculation, we used that all three mooring pairs are statistically indistinguishable and that orthogonal velocity components are uncorrelated per the isotropy assumption. In the third line, $\mathbf{t}_{i}^{\prime}$ and $\mathbf{n}_{i}^{\prime}$ are the transverse and longitudinal unit vectors with respect to the mooring pair $(1,2)$ (Fig. 4b). They are related to the triangle's tangent vectors by $\mathbf{t}_{1}=\cos \alpha \mathbf{t}_{1}^{\prime}-\sin \alpha \mathbf{n}_{1}^{\prime}$ and $\mathbf{t}_{2}=\cos \alpha \mathbf{t}_{2}^{\prime}+\sin \alpha \mathbf{n}_{2}^{\prime}$. The angle $\alpha=\pi / 6$ is half of the interior angle of the mooring triangle. The formula for the divergent part follows by replacing the $\mathbf{t}_{i}$ by the outward normal unit vectors $\mathbf{n}_{i}$ (Fig. 4b).

Notably, the triplet statistics in (19) can be calculated from a single mooring pair-there is no need for an actual mooring triplet arranged in an equilateral triangle. This is a consequence of the homogeneity and isotropy assumptions, in combination with the fact that all mooring pairs in the triangle have the same separation $r$.

\section{c. Linear combination of weighting kernels}

We begin by writing the Helmholtz decomposition (7) in spectral space:

$$
\begin{aligned}
& |\hat{u}(\mathbf{k})|^{2}=l^{2}|\hat{\psi}(\mathbf{k})|^{2}+k^{2}|\hat{\phi}(\mathbf{k})|^{2}-2 k l \operatorname{Re} \hat{\psi}^{*}(\mathbf{k}) \hat{\phi}(\mathbf{k}), \\
& |\hat{v}(\mathbf{k})|^{2}=k^{2}|\hat{\psi}(\mathbf{k})|^{2}+l^{2}|\hat{\phi}(\mathbf{k})|^{2}-2 k l \operatorname{Re} \hat{\psi}^{*}(\mathbf{k}) \hat{\phi}(\mathbf{k}),
\end{aligned}
$$

where $k=\mathbf{k} \cdot \mathbf{r} / r=\kappa \cos \theta$ is the longitudinal component of the wavevector, $l=\mathbf{k} \cdot(\mathbf{z} \times \mathbf{r}) / r=\kappa \sin \theta$ is the transverse component of the wavevector, and $\theta$ is the angle between $\mathbf{r}$ and the wavevector $\mathbf{k}$. Then the longitudinal and transverse structure functions can be written as (the cross term drops out by symmetry if isotropy is assumed)

$$
\begin{aligned}
\Delta^{u}(r)= & \frac{1}{2 \pi} \int_{0}^{\infty} \int_{-\pi}^{\pi} \kappa^{2}\left[\sin ^{2} \theta\left\langle|\hat{\psi}(\kappa)|^{2}\right\rangle+\cos ^{2} \theta\left\langle|\hat{\phi}(\kappa)|^{2}\right\rangle\right] \\
& \times\left(1-e^{i \kappa r \cos \theta}\right) d \theta d \kappa
\end{aligned}
$$

$$
\begin{aligned}
\Delta^{v}(r)= & \frac{1}{2 \pi} \int_{0}^{\infty} \int_{-\pi}^{\pi} \kappa^{2}\left[\cos ^{2} \theta\left\langle|\hat{\psi}(\kappa)|^{2}\right\rangle+\sin ^{2} \theta\left\langle|\hat{\phi}(\kappa)|^{2}\right\rangle\right] \\
& \times\left(1-e^{i \kappa r \cos \theta}\right) d \theta d \kappa
\end{aligned}
$$

The azimuthal integrations evaluate to

$$
\begin{aligned}
& \Delta^{u}(r)=\int_{0}^{\infty} \kappa^{2}\left[\left\langle|\hat{\psi}(\kappa)|^{2}\right\rangle F_{1}(\kappa r)+\left\langle|\hat{\phi}(\kappa)|^{2}\right\rangle F_{2}(\kappa r)\right] d \kappa, \\
& \Delta^{v}(r)=\int_{0}^{\infty} \kappa^{2}\left[\left\langle|\hat{\psi}(\kappa)|^{2}\right\rangle F_{2}(\kappa r)+\left\langle|\hat{\phi}(\kappa)|^{2}\right\rangle F_{1}(\kappa r)\right] d \kappa,
\end{aligned}
$$

where the weighting kernels are

$$
F_{1}(\xi)=\frac{1}{2}-\frac{J_{1}(\xi)}{\xi} \quad \text { and } \quad F_{2}(\xi)=\frac{1}{2}-\frac{J_{1}(\xi)}{\xi}+J_{2}(\xi)
$$

The reversed kernels in (24) and (25) confirm that we can extract information on the rotational and divergent components of the flow from the longitudinal and transverse structure functions (Fig. 6a). The longitudinal and transverse structure functions sample different parts of the rotational and divergent components of the flow. Ideally, we would find combinations of the kernels that isolate

$$
\begin{aligned}
& \Delta_{\text {rot }}(r)=\int_{0}^{\infty} \kappa^{2}\left\langle|\hat{\psi}(\kappa)|^{2}\right\rangle F_{0}(\kappa r) d \kappa, \\
& \Delta_{\text {div }}(r)=\int_{0}^{\infty} \kappa^{2}\left\langle|\hat{\phi}(\kappa)|^{2}\right\rangle F_{0}(\kappa r) d \kappa .
\end{aligned}
$$

The exact Helmholtz decomposition (8) and (9) achieves just that: taking the difference of (24) and (25) isolates the $J_{2}(\kappa r)$ term, division by $r$ and integration over $r$ yields $F_{1}(\kappa r)$ for the kernels, and combining that back with the respective structure functions cancels one contribution and consolidates the other to an $F_{0}(\kappa r)$ kernel.

If structure functions are available at a single separation $r$ only, the weighting kernels in (24) and (25) cannot be combined to exactly isolate the rotational and divergent components. One arrives at the approximate single-separation decomposition (10), however, if one combines the kernels optimally in the $\kappa r \ll 1$ limit. Using Taylor series expansions around $\xi \equiv \kappa r=0$, one finds that the kernel functions satisfy

$$
\begin{aligned}
& F_{0}(\xi)=\frac{\xi^{2}}{4}+\mathscr{O}\left(\xi^{4}\right), \quad F_{1}(\xi)=\frac{\xi^{2}}{16}+\mathscr{O}\left(\xi^{4}\right), \\
& F_{2}(\xi)=\frac{3 \xi^{2}}{16}+\mathscr{O}\left(\xi^{4}\right) .
\end{aligned}
$$



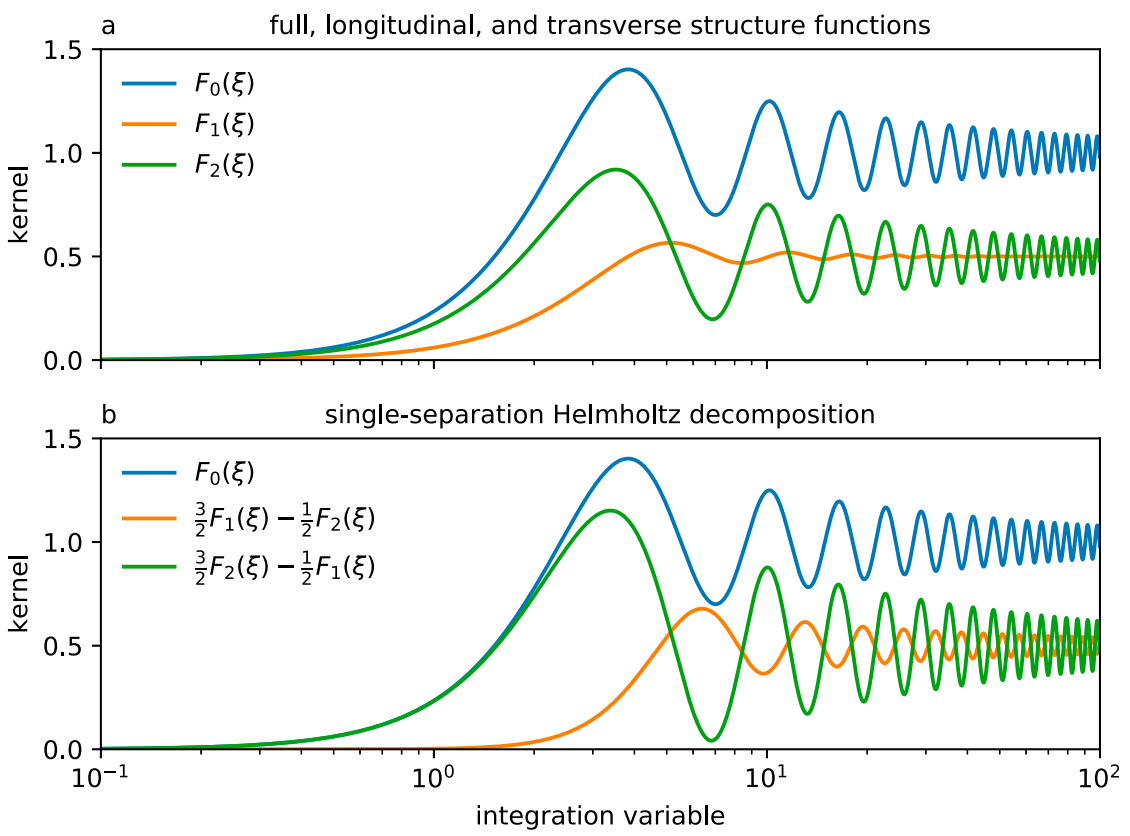

FIG. 6. Integration kernels with which the wavenumber spectra are sampled. (a) Integration kernels for the full, longitudinal, and transverse structure functions for a flow that is purely rotational. (b) Integration kernels for the rotational and divergent components of the flow in the diagnosis of the rotational component. The divergent component spills into the diagnosed rotational component for $\xi \gtrsim \pi$.

It is straightforward to find linear combinations that asymptotically match the kernels $F_{0}(\xi)$ and 0 , that is, that isolate the rotational or divergent components (Fig. 6b):

$$
\begin{aligned}
& \frac{3}{2} F_{2}(\xi)-\frac{1}{2} F_{1}(\xi)=F_{0}(\xi)+\mathcal{O}\left(\xi^{4}\right), \\
& \frac{3}{2} F_{1}(\xi)-\frac{1}{2} F_{2}(\xi)=\mathscr{O}\left(\xi^{4}\right) .
\end{aligned}
$$

These linear combinations yield (10).

\section{d. Accuracy}

The single-separation Helmholtz decomposition (10) is accurate in the limit of large-scale dominance (i.e., spectra falling off more steeply than $\kappa^{-3}$ ). The formulas have no distinguishing power if flow is decorrelated across the separation $r$ (i.e., if the flow is dominated by scales smaller than $r$ ). In that latter case, the formulas assign $1 / 2$ of the energy to each component even if the flow is purely rotational or purely divergent.

To get a sense for the accuracy of the method in the local regime, that is, between the two limits above, consider a purely rotational flow that at high wavenumbers follows a power-law spectrum $\kappa^{-n}$ with $1<n<$ 3. At small separations, $\Delta^{u}(r)$ and $\Delta^{v}(r)$ follow a power law $r^{n-1}$ (e.g., Babiano et al. 1985) and $\Delta^{v}(r)=n \Delta^{u}(r)$ (e.g., Charney 1971). The rotational part is then diagnosed to be $(3 n-1) / 2(n+1)$ of the total energy, and the remainder is assigned erroneously to the divergent part (Fig. 7). For $n=2$, for example, the formulas (10) assign $5 / 6$ of the energy to the rotational component and $1 / 6$ of the energy to the divergent component. The fractional error assumes the nonlocal limits 1 and $1 / 2$ for $n=3$ and $n=1$, respectively.

The formulas (10) are thus accurate in the large-scale limit, still give qualitatively useful results in the local regime, and have no distinguishing power in the smallscale limit. These properties can be intuited from the derivation as a diagnostic from a mooring triangle: the triangle perfectly resolves flow dominated by scales larger than the triangle's size, and the triangle has no chance of distinguishing between rotational and divergent flow if the dominant scales are smaller than the triangle's size. For scales similar to the triangle's size, the triangle yields some information on the Helmholtz properties of the flow, but the diagnostics are not perfect.

\section{e. Rossby number}

The single-separation Helmholtz decomposition also allows us to define a scale-dependent Rossby number. To obtain the right normalization constant, we 


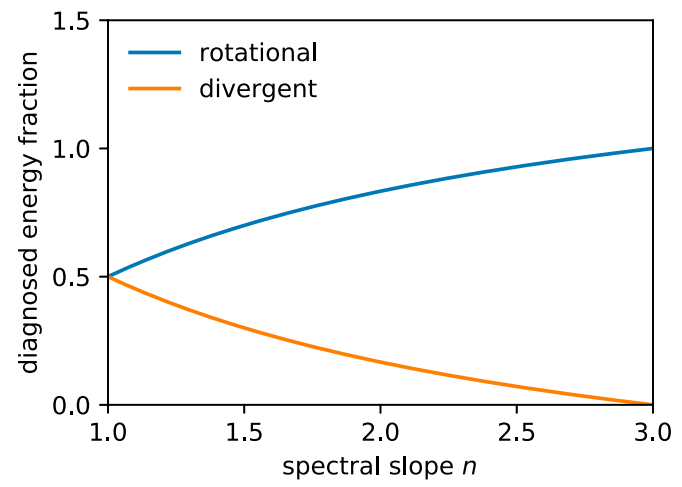

FIG. 7. Accuracy of the single-separation Helmholtz decomposition. Shown are the diagnosed rotational and divergent fractions of the total energy, assuming that the actual flow is purely rotational and has an energy spectrum that follows a power law $\kappa^{-n}$.

again consider a triangular mooring array, which suggests how to calculate the vorticity (or analogously divergence):

$$
\begin{aligned}
\zeta(r) & =\frac{1}{\pi R^{2}} \int \nabla \times \mathbf{u} d^{2} \mathbf{x}=\frac{1}{\pi R^{2}} \oint \mathbf{u} \cdot \mathbf{t} d s \approx \frac{2}{3 R} \sum_{i=1}^{3} \mathbf{u}_{i} \cdot \mathbf{t}_{i} \\
& =\frac{2}{r} u_{\mathrm{rot}},
\end{aligned}
$$

where the first integration is over the disk enclosed by the circumcircle of the mooring triangle (radius $R=r / \sqrt{3}$ ), and the second integration is along the circumcircle itself. This tells us that

$$
\left\langle\zeta^{2}(r)\right\rangle=\frac{4}{r^{2}} \Delta_{\text {rot }}(r),
$$

and we consequently define a scale-dependent Rossby number as

$$
\operatorname{Ro}(r)=\frac{\left\langle\zeta^{2}(r)\right\rangle^{1 / 2}}{f}=\frac{2}{f r}\left[\Delta_{\mathrm{rot}}(r)\right]^{1 / 2}
$$

For frequency-resolved data, $\Delta_{\text {rot }}(r, \omega)$ is a spectral density in frequency space and carries an additional time dimension. We therefore define the scale- and frequency-dependent Rossby number as

$$
\operatorname{Ro}(r, \omega)=\frac{2}{f r}\left[\omega \Delta_{\text {rot }}(r, \omega)\right]^{1 / 2} .
$$

We will estimate this from the mooring data.

This Rossby number characterizes the importance of inertial terms at a wavelength $2 r$ and a period of $2 \pi / \omega$. A small Rossby number indicates dynamics that are to leading order geostrophic and thus horizontally nondivergent. We will see below that we consistently diagnose the observed submesoscale flow to be dominated by the rotational component and to have a Rossby number smaller than one all the way down to the smallest scales sampled by the mooring array.

\section{Results}

\section{a. Frequency-resolved structure functions}

\section{1) Shallow Wintertime Statistics}

We begin by discussing the frequency spectrum $\left\langle|\hat{u}(\omega)|^{2}+|\hat{v}(\omega)|^{2}\right\rangle / 2$ and the frequency-resolved structure function $\left[\Delta^{u}(r, \omega)+\Delta^{v}(r, \omega)\right] / 2$ calculated from the instruments near 50-m depth and for the winter period. We subsequently contrast these results with the other seasons and the data from near 500-m depth. The statistics are calculated the same way as described above for the model simulation.

The frequency spectrum has familiar features (Fig. 8b; e.g., Ferrari and Wunsch 2009). There is broadband energy at subinertial frequencies, a peak at the inertial frequency, a peak at the semidiurnal tidal frequency, and broadband energy at supertidal frequencies. The spectrum flattens out at frequencies larger than $2 \times 10^{-4} \mathrm{cps}$, which we attribute to instrument noise. The broadband subinertial energy falls off slowly with frequency, roughly like $\omega^{-1}$. Within the bounds of the spectral estimation error, the statistics are homogeneous and isotropic except at the semidiurnal tidal frequency, where anisotropy can be detected (not shown).

The frequency-resolved structure functions now allow us to assign spatial scales to the frequencies covered by the frequency spectrum. At the lowest frequencies, the frequency spectrum dominates over the frequency-resolved structure functions at all separations, suggesting that the energy is dominated by scales larger than the largest sampled scale $2 r=$ $37.4 \mathrm{~km}$. As the frequency increases, the frequencyresolved structure functions for the larger separations $(r=7.8-18.7 \mathrm{~km})$ successively converge to the frequency spectrum within the subinertial range. This indicates a successive decrease in spatial scale that accompanies the decrease in time scales, as expected for a turbulent flow. The flow just below the inertial frequency is dominated by a scale of about $2 r=$ $10 \mathrm{~km}$ - the flow is decorrelated at a separation $r=$ $7.8 \mathrm{~km}$ but correlated at a separation $r=3.1 \mathrm{~km}$. Submesoscale turbulence at scales larger than $10 \mathrm{~km}$ is thus associated with subinertial frequencies. Submesoscale turbulence at scales smaller than $10 \mathrm{~km}$ appear to have 

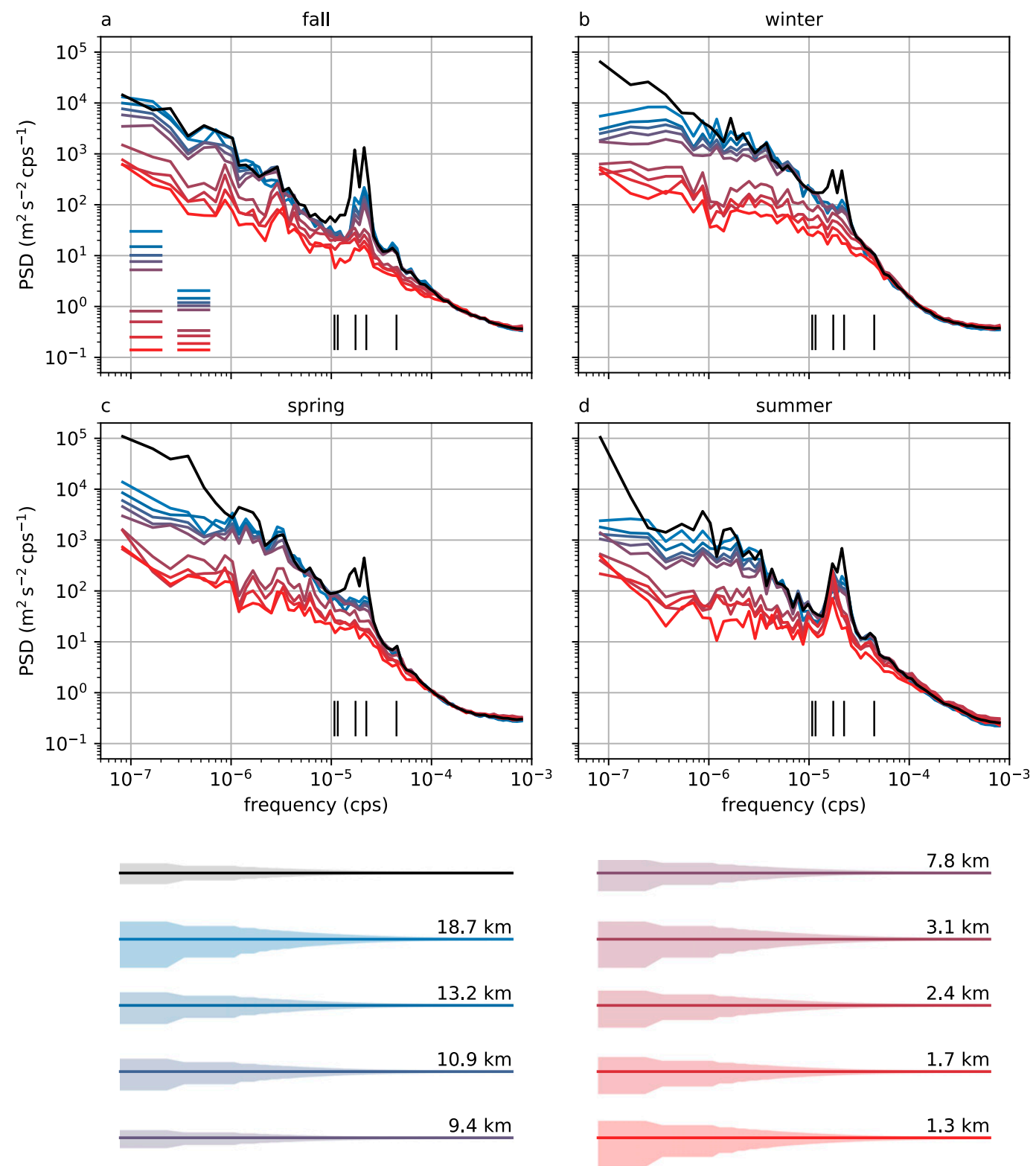

FIG. 8. Frequency-resolved structure functions estimated for the four seasons from the OSMOSIS array at 50-m depth. The colored lines show the frequency-resolved structure functions for the separations $r$ given in the legend, and the black lines show the kinetic energy frequency spectra. The formal error bars are indicated below the four panels and vary by separation. Short black vertical lines mark the two lunar diurnal frequencies, the inertial frequency, the lunar semidiurnal frequency, and the first harmonic of the lunar semidiurnal frequency. For reference, the short horizontal colored lines in (a) show the spacing of structure functions that vary with $r^{2}$ and $r^{1}$.

Eulerian time scales comparable to or shorter than the inertial period.

At the inertial frequency, the frequency spectrum again strongly dominates over the frequency-resolved structure functions at all separations, indicating that these near-inertial motions have large spatial scales. The frequency-resolved structure functions do not show a distinct signal at the inertial frequency, suggesting that at the captured scales dynamics other than near-inertial motion dominate.

At the semidiurnal tidal frequency, the frequency spectrum also dominates over the frequency-resolved structure functions at all separations, suggesting the signal is dominated by tidal motion that has 
wavelengths larger than the largest scale sampled by the array: either external tides or low-mode internal tides. The frequency-resolved structure functions at the larger separations have a small tidal peak, suggesting that tidal waves still dominate at these spatial scales. At the smaller separations, there is no discernible tidal signal.

In the supertidal frequency band, the frequencyresolved structure functions match the frequency spectrum for the larger separations and successively converge onto the frequency spectrum for the smaller separations. This indicates that this frequency band is dominated by spatial scales of a few kilometers at the lower frequencies and of order $1 \mathrm{~km}$ or smaller at the higher frequencies. At $\omega=10^{-4} \mathrm{cps}$, the flow is completely decorrelated across all moorings.

As justified further below, we interpret the flows at $1-10 \mathrm{~km}$ in the winter mixed layer as an extension of balanced submesoscale turbulence into the superinertial range. At scales smaller than $1 \mathrm{~km}$, atmospherically forced three-dimensional turbulence likely makes a contribution to the frequency spectrum (cf. Evans et al. 2018), but the mooring array cannot resolve such small lateral scales.

\section{2) SEAsonality}

The frequency statistics undergo substantial seasonality (Figs. 8a-d). At $\omega=10^{-5} \mathrm{cps}$, that is, a period of about 1 day, the frequency spectrum is almost an order of magnitude larger in winter than in summer. This frequency remains dominated by a spatial scale around $10 \mathrm{~km}$ throughout the year, indicating that this seasonality in the frequency spectrum is a seasonality in submesoscale turbulence.

At lower frequencies, the seasonality is less pronounced. At $\omega=10^{-6} \mathrm{cps}$, that is, a period of about 10 days, there is little seasonality. At even lower frequencies, there appears to be some seasonality, but the error bars are large. At these periods, there are few degrees of freedom because the segments are only 140 days long and the spatial scale of the flow is large, such that the motion across the moorings is correlated. The formal error bars on the frequency spectrum are underestimates of the true uncertainty because they assume decorrelated signals at all moorings.

The near-inertial motion also undergoes a seasonal cycle. The peak changes its amplitude somewhat between the seasons, but most notable is the seasonality at small scales. While there is no discernible inertial peak at the sampled separations in winter or spring, there are clear peaks at all separations in summer and fall. A plausible explanation would be that nonlinear submesoscale turbulence dominates at the inertial frequency in winter and spring, drowning out any signal from inertial motion. The turbulent motion generates a broadband signal in both wavenumber and frequency space, and no peak occurs in the frequency statistics. In summer and fall, instead, nearinertial waves that are linear to leading order appear to dominate. In summer, in particular, the spatial scales of near-inertial waves are remarkably small, with a significant part of the variance at separations of order $1 \mathrm{~km}$. We speculate that the increased Burger number of the seasonal thermocline may enhance the dispersion of near-inertial waves by geostrophic flow, facilitating the transfer of near-inertial energy to small scales (e.g., Young and Ben Jelloul 1997; Xie and Vanneste 2015; Thomas et al. 2017; Rocha et al. 2018).

There is some seasonality in the semidiurnal tidal peak, with the highest energy occurring in fall. In all seasons, there are discernible tidal peaks in the frequency-resolved structure functions at the larger separations but not the smaller separations.

The supertidal frequencies display some seasonality as well. The broadband energy level is somewhat reduced outside of winter, letting a minor peak at the tidal harmonic at $\omega=4.5 \times 10^{-5} \mathrm{cps}$ emerge in spring, summer, and fall.

\section{3) Pressure gradients}

To further investigate the distribution of energy near the inertial frequency, we now consider the power spectra of pressure gradients as inferred from linear dynamics:

$$
i \omega \tilde{u}-f \tilde{v}=-\tilde{p}_{x} \quad \text { and } \quad i \omega \tilde{v}+f \tilde{u}=-\tilde{p}_{y} .
$$

We calculate the frequency spectra $\left\langle\left|\tilde{p}_{x}(\omega)\right|^{2}+\right.$ $\left.\left|\tilde{p}_{y}(\omega)\right|^{2}\right\rangle / 2 f^{2}$ and the frequency-resolved structure functions $\left[\Delta^{p_{x}}(r, \omega)+\Delta^{p_{y}}(r, \omega)\right] / 2 f^{2}$. We include the $f^{-2}$ normalization such that these pressure gradient statistics match the velocity statistics for geostrophic flow. For strongly superinertial flow, these pressure gradient statistics are equal to $\omega^{2} / f^{2}$ times the velocity statistics. But the pressure gradient statistics reveal new information around the inertial frequency because they filter out linear inertial motion, which consists of a balance between the tendency and Coriolis terms and produces no pressure gradients. Any remaining signal at the inertial frequency must thus be due to nonlinear dynamics. The inferred pressure gradients are then of course not equal to the true pressure gradients but instead include contributions from the nonlinear terms. 

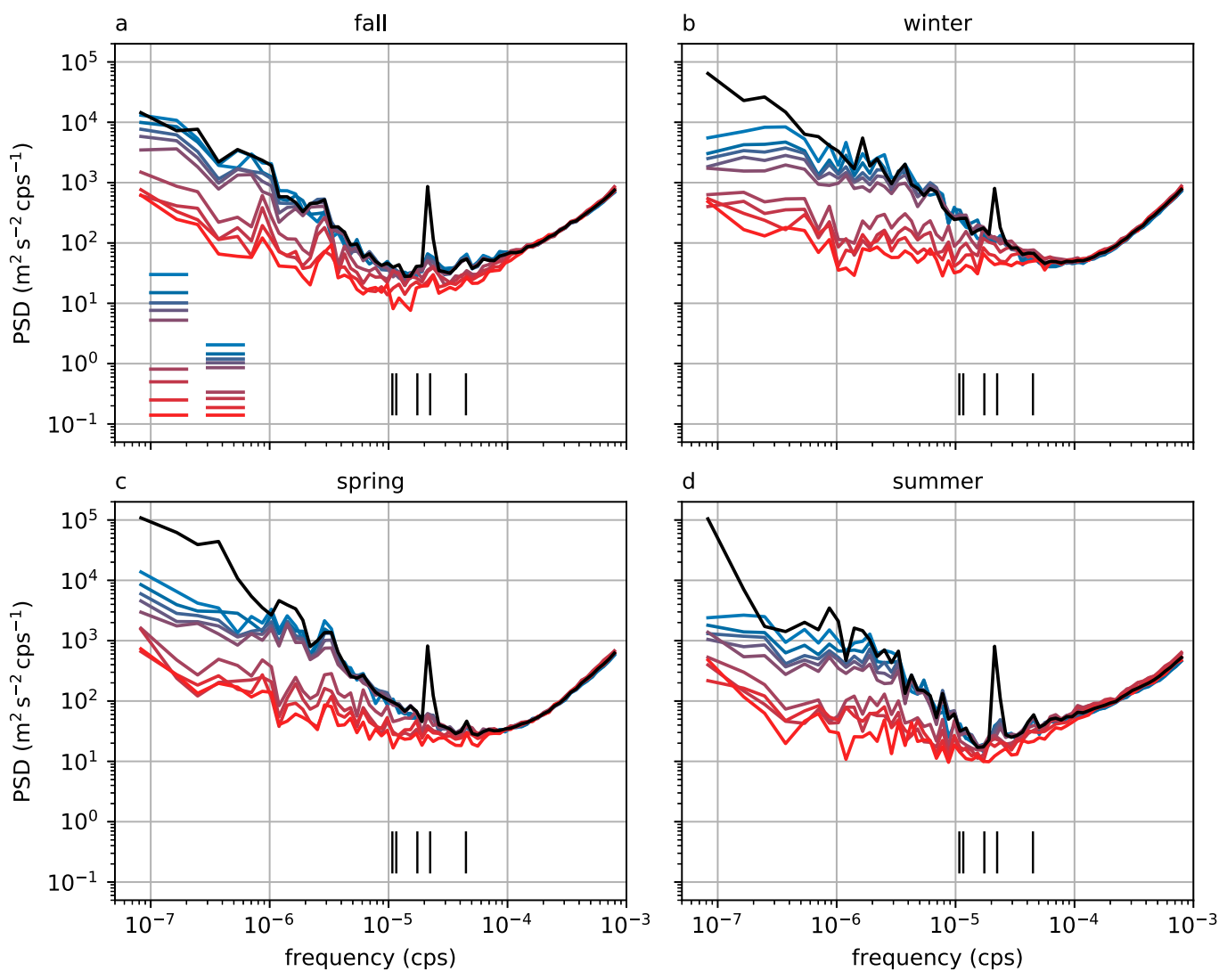

FIG. 9. Frequency-resolved structure functions for pressure gradients, estimated for the four seasons from the OSMOSIS array at 50-m depth. All lines and error bars are the same as in Fig. 8.

These pressure gradient statistics cleanly remove the inertial peak (Fig. 9). This confirms that the inertial peak is due to linear dynamics, and it allows us to "see through" the inertial peak. In winter, where there is no discernible inertial peak in the frequencyresolved structure functions at any separation, the pressure gradient statistics confirm that this broadband energy is due to nonlinear dynamics, that is, submesoscale turbulence. This supports the picture that submesoscale turbulence extends to superinertial Eulerian frequencies, in this case for spatial scales smaller than about $10 \mathrm{~km}$. These pressure gradient statistics also confirm the seasonal cycle in this strongly nonlinear submesoscale turbulence with variance levels that differ by an order of magnitude between winter and summer.

In contrast to linear inertial waves, linear tidal waves are associated with a pressure signal. A prominent peak thus remains in the pressure gradient statistics at the semidiurnal frequency. Interestingly, there is less seasonality in this peak than in the velocity statistics, possibly because seasonally variable near-inertial or submesoscale energy leaks into the tidal frequency in the velocity statistics but not in the pressure gradient statistics.

\section{4) DEEP DATA}

At 500-m depth, the general shape of the frequency spectra and the frequency-resolved structure functions is similar to that of the statistics at $50-\mathrm{m}$ depth (Fig. 10). At low frequencies, the energy levels at $500 \mathrm{~m}$ are similar to those at $50 \mathrm{~m}$, as expected for thermocline-spanning mesoscale eddies. At higher frequencies, which are associated with smaller scales, the energy is reduced by about an order of magnitude compared to $50-\mathrm{m}$ depth. The frequency spectra are substantially steeper, indicating that small-scale energy is more surface trapped.

The subinertial energy around $\omega=10^{-5} \mathrm{cps}$ still undergoes a substantial seasonal cycle. The difference between winter and summer is somewhat muted, but the minimum now occurs in fall and is still about an order of magnitude lower than in winter. While $500 \mathrm{~m}$ does not fall into the mixed layer according to common definitions, it still is in the weakly stratified subpolar mode water layer above the main thermocline 

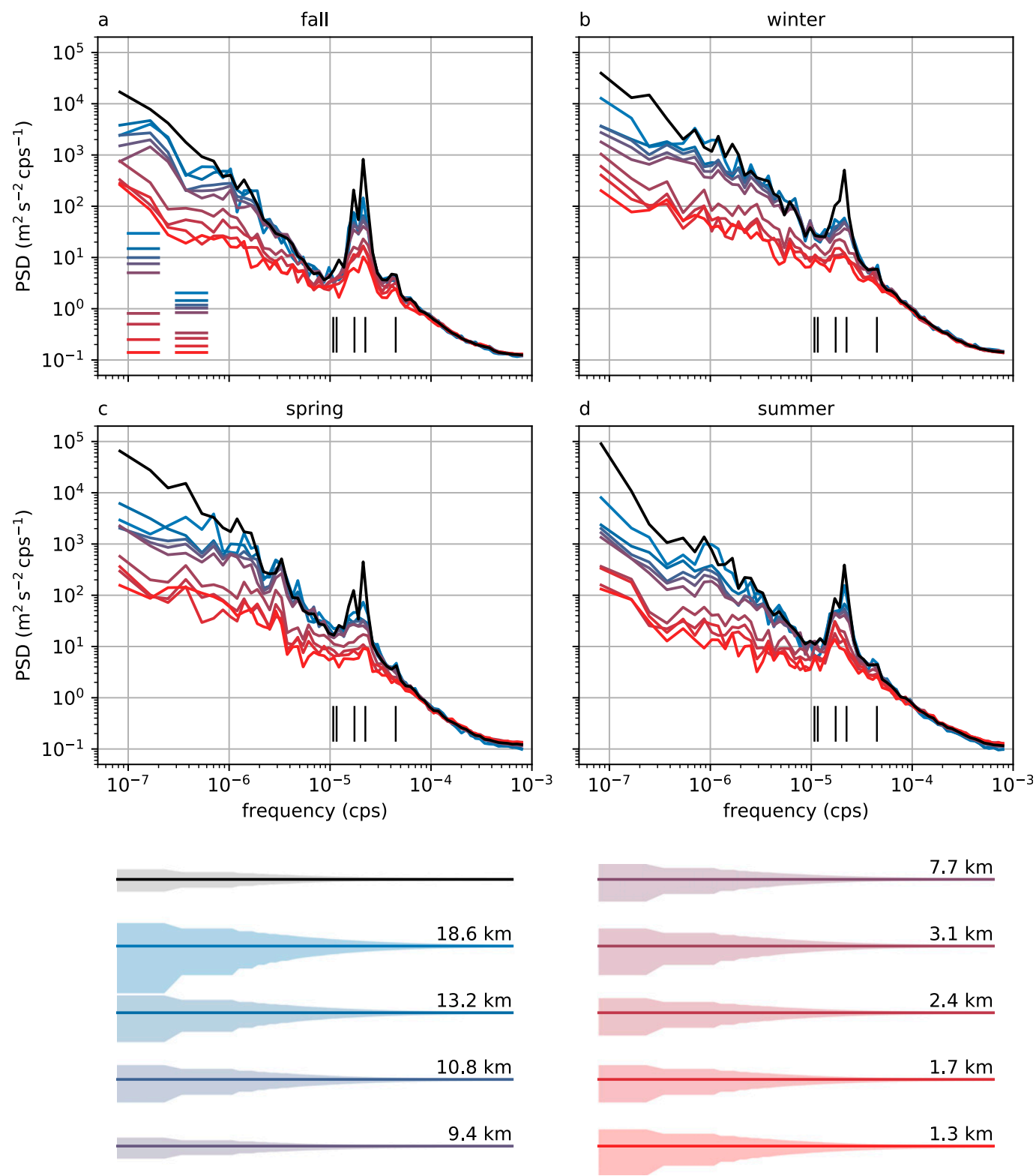

FIG. 10. Frequency-resolved structure functions estimated for the four seasons from the OSMOSIS array at 500-m depth. The presentation is the same as in Fig. 8.

(Fig. 2c). Unlike strongly stratified thermocline water, this layer therefore does experience a seasonal energization of submesoscales (Erickson et al. 2020).

\section{b. Helmholtz decomposition}

To further elucidate the submesoscale dynamics, we now apply the single-separation Helmholtz decomposition introduced in section 4 to the wintertime mooring data at 50-m depth (Fig. 11). For every separation $r$, we compare the frequency spectrum, the frequency-resolved structure function $\left[\Delta^{u}(r, \omega)+\right.$ $\left.\Delta^{v}(r, \omega)\right] / 2$, and the diagnosed rotational component
$\Delta_{\text {rot }}(r, \omega) / 2$. It is important to remember here that the single-separation Helmholtz decomposition can only distinguish between rotational and divergent flow if the flow is dominated by scales comparable to or larger than $r$. This is not the case if the frequency-resolved structure function matches the frequency spectrum, that is, if the flow sampled at moorings separated by a distance $r$ is decorrelated. In such a case with smallscale dominance, the single-separation Helmholtz decomposition assigns half the energy to the rotational component, irrespective of the actual rotational content of the flow. 

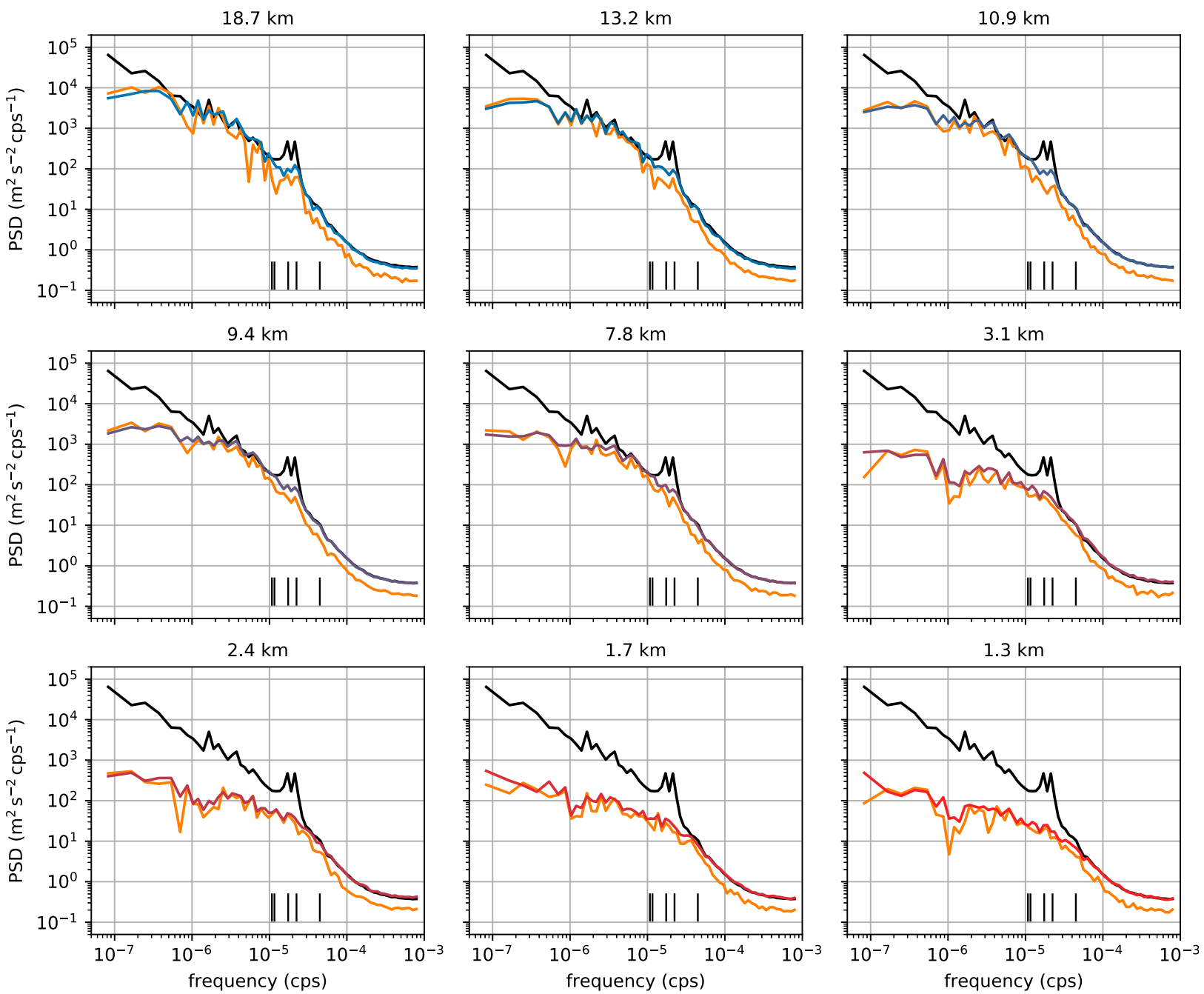

FIG. 11. Single-separation Helmholtz decomposition of the wintertime data at 50-m depth. Each panel corresponds to the separation $r$ given in the title. For reference, the kinetic energy frequency spectrum (black line) is shown in every panel. For every separation, the frequency-resolved structure function (blue to red lines) are compared to the diagnosed rotational component (orange lines). The reference frequencies marked by vertical black lines are the same as in Fig. 8.

Across almost all separations and frequencies, the Helmholtz decomposition indicates rotational dominance where it has distinguishing power. Wherever the frequency-resolved structure function falls below the frequency spectrum, the diagnosed rotational component makes up a large fraction of the total energy. Where the frequency-resolved structure functions converge to the frequency spectrum, the rotational component drops to one-half of the energy, as expected.

There are only a few exceptions to this general picture. At low frequencies, where estimation errors are large, there are occasional fluctuations of the rotational component away from the corresponding frequencyresolved structure function. Similarly, the diagnosed rotational component sometimes makes up a fraction larger than one-half where the frequency-resolved structure functions match the frequency spectrum and the decomposition has no distinguishing power (e.g., at $r=13.2 \mathrm{~km}$ and $\omega \approx 5 \times 10^{-6} \mathrm{cps}$ ). At the inertial and tidal frequencies, the rotational component drops to half or even less of the frequencyresolved structure function for the larger separations. The most likely reason for this is that submesoscale turbulence at these frequencies and spatial scales is decorrelated (cf. Fig. 9), but there could also be a nonnegligible contribution from linear waves with a substantial divergent component. This feature disappears at smaller separation, where submesoscale turbulence is correlated across mooring pairs. 
In summary, this decomposition suggests that the energetic submesoscale turbulence that dominates the spatial scales sampled by the mooring array is largely rotational, that is, horizontally nondivergent. This is the case even at inertial and slightly superinertial frequencies, where the flow is dominated by spatial scales of a few kilometers.

\section{c. Rossby numbers}

To further assess the dynamics of the energetic wintertime submesoscale turbulence, we now calculate the frequency- and scale-dependent Rossby number defined in (34) for the winter data at 50-m depth (Fig. 12). For every separation $r$, the Rossby number first increases with frequency, reaches a maximum at intermediate frequencies, and then decreases. At frequencies larger than $\omega=2 \times 10^{-4} \mathrm{cps}$ the Rossby numbers for all separations increase again, but this frequency range is likely contaminated by instrument noise and should be ignored. The peak Rossby number increases from about 0.1 for $r=18.7 \mathrm{~km}$, reached around $\omega=2 \times 10^{-6} \mathrm{cps}$, to about 0.5 for $r=1.3 \mathrm{~km}$, reached around the inertial frequency. This peak Rossby number at the smallest accessible scales is roughly consistent with the Rossby number that Buckingham et al. (2016) estimated from triangles formed by the inner and central moorings, that is, on the smallest scales considered here (Fig. 3).

The increase of peak Rossby number with decreasing separation $r$ is expected for energetic submesoscale turbulence. If the wavenumber spectrum falls off like $k^{-2}$, the scale-dependent Rossby number increases with $k^{1 / 2}$ or $r^{-1 / 2}$. The increase from 0.1 to 0.5 is roughly consistent with the expected factor $(18.7 \mathrm{~km} / 1.3 \mathrm{~km})^{1 / 2}=4$.

If this scaling continues to hold at smaller scales, the Rossby number reaches one around $r=300 \mathrm{~m}$. This is somewhat smaller than what Callies et al. (2015) inferred for the western subtropical North Atlantic, not surprisingly given that the mesoscale and submesoscale energy levels are substantially lower in the OSMOSIS region (Thompson et al. 2016).

A Rossby number of 0.5 at the smallest scales captured by the mooring is also consistent with the diagnosed rotational dominance in submesoscale turbulence. While at such a Rossby number advective nonlinearities are starting to become important, the flow is expected to retain a rotational dominance until the Rossby number becomes one. Given the level of accuracy that the single-separation Helmholtz decomposition can diagnose the rotational component with, the two diagnostics agree in their characterization of

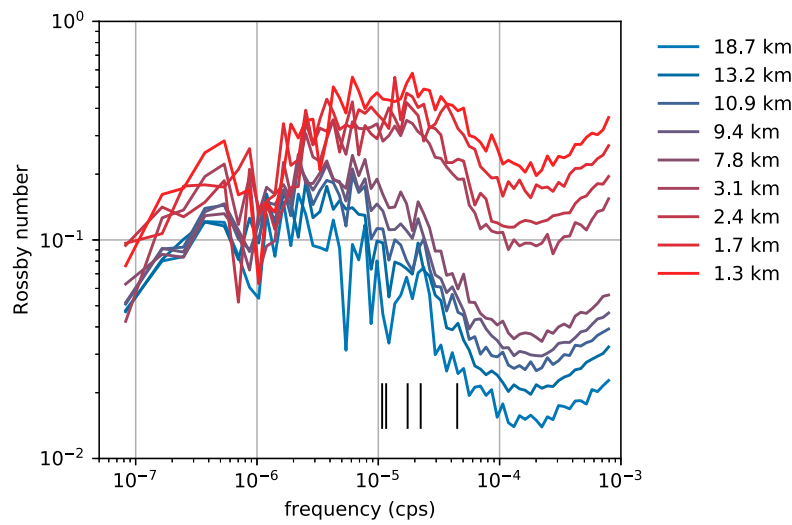

FIG. 12. Scale- and frequency-dependent Rossby number for the wintertime data at 50-m depth. The separations $r$ are given in the legend. The reference frequencies marked by vertical black lines are the same as in Fig. 8 . The data beyond $\omega=2 \times 10^{-4} \mathrm{cps}$ are dominated by noise and should be ignored.

the flow as somewhat but not entirely geostrophically dominated.

It is important to emphasize that the Rossby number at $r=1.3 \mathrm{~km}$ peaks at the inertial frequency but is not one. This means that the advective time scale diagnosed with the Rossby number is smaller than the Eulerian time scale diagnosed with the frequency decomposition. That the flow evolves on a dominant time scale comparable to the inertial period in an Eulerian frame therefore does not necessarily imply that the nonlinear terms in the momentum equation have the same magnitude as the Coriolis and pressure gradient terms. In other words: the Lagrangian evolution may still be subinertial, even if the Eulerian evolution is inertial or superinertial.

\section{Conclusions}

Our analysis of the OSMOSIS mooring array data confirms the energization of the submesoscale range in winter. We can now unambiguously attribute this seasonal cycle to balanced submesoscale turbulence, whose energy level frequency-resolved structure functions show to vary by about an order of magnitude between winter and the rest of the year. This supports the dynamical interpretation previously gleaned from wavenumber spectra and their dynamical decomposition, which is that balanced flows induced by mixed layer instabilities produce the wintertime energization.

Furthermore, the OSMOSIS array allows us to assign time scales to the energetic wintertime submesoscale turbulence. We find that wavelengths of order $10 \mathrm{~km}$ correspond to time scales of order 1 day, 
suggesting that submesoscale turbulence at scales smaller than $10 \mathrm{~km}$ evolves on time scales similar to or shorter than the inertial period. At the same time, we infer from an abridged Helmholtz decomposition that submesoscale turbulence is largely horizontally nondivergent across the observed scales, and we find that the scale-dependent Rossby number does not exceed 0.5 down to spatial scales of $2.6 \mathrm{~km}$. This suggests that while Eulerian time scales are superinertial at spatial scales of about $10 \mathrm{~km}$ and smaller, the Lagrangian evolution is likely subinertial down to subkilometer scales. This is broadly consistent with previous estimates of scale-dependent Rossby numbers from observed wavenumber spectra (Callies et al. 2015) and wavenumber-frequency spectra from numerical simulations (Rocha et al. 2016; Qiu et al. 2018; Torres et al. 2018).

These findings suggest that a description of submesoscale turbulence based on subinertial dynamics is at least not qualitatively misleading down to scales of a few kilometers. It should be kept in mind, however, that our statistics only speak to average conditions. Especially in an intermittent flow field, strongly ageostrophic dynamics can still play a role locally. For example, our statistics do not deny the importance of accelerated sharpening of submesoscale buoyancy gradients produced by mixed layer instabilities into sharp fronts. Yet, our statistics suggest that, on average, horizontal divergence becomes comparable to the vertical vorticity only at scales of order $1 \mathrm{~km}$ and smaller.

Acknowledgments. The OSMOSIS project was funded by grants from the Natural Environmental Research Council (NE/1019999/1 and NE/101993X/1). We thank Andrew Thompson for providing us with the glider data used in Fig. 2c. JC was partially supported by NSF Grant OCE-1924354. RB was supported by ONR-N000141812697, NSF Grant OCE-1851397, and Israeli Science foundation Grant 1736/18. ACNG acknowledges the support of the Royal Society and the Wolfson Foundation.

\section{APPENDIX}

\section{Mooring Knockdown}

In May 2013, strong currents knocked down the uppermost current meters on the OSMOSIS moorings by more than $200 \mathrm{~m}$ in depth (Fig. A1). While such a large knockdown is rare in the year-long deployment-the root-mean-square knockdown is $25 \mathrm{~m}$ for the instruments at $50-\mathrm{m}$ nominal depth and $21 \mathrm{~m}$ for the instruments at 500-m nominal depth (Figs. A1a,b) - it does create concern about the reliability of the recorded currents.

To estimate the velocity error, we assume (pessimistically) that the moorings are stiff pendulums anchored at the seafloor at depth $H$. Then the angle $\theta$ between the mooring line and the true vertical can be determined by the instrument depth $h$ inferred from the pressure sensor on the current meter. If $h_{0}$ denotes the depth of the instrument when the mooring is upright, then

$$
\theta=\arccos \frac{H-h}{H-h_{0}} .
$$

The azimuthal velocity of the instrument is then $\left(H-h_{0}\right) d \theta / d t$. For small angles $\theta$, the azimuthal motion is roughly horizontal, so we will consider it as an estimate for the horizontal velocity error due to the mooring motion.

The spectrum of the azimuthal mooring motion $\left(H-h_{0}\right)^{2} \omega^{2}\left\langle|\hat{\theta}(\omega)|^{2}\right\rangle$ is well below the kinetic energy spectrum inferred from the recorded currents (Fig. A1c). These frequency spectra are estimated from the full mooring record, with the same spectral estimation procedure as described in the main text. The most prominent azimuthal mooring motion occurs at the semidiurnal tidal frequency, presumably because the periodic nature of the tidal currents more effectively moves the mooring line than a quasi-random turbulent flow. Even at its extreme (at the first harmonic of the semidiurnal lunar tide), the spectrum of the azimuthal mooring motion is a factor 20 below the kinetic energy spectrum. This suggests that our spectral estimates are robust at roughly a 5\% level-and probably better because the moorings are not stiff pendulums.

We do not have enough information to estimate the longitudinal velocity $\left(H-h_{0}\right) \sin \theta d \lambda / d t$, where $\lambda$ denotes the longitudinal angle of the mooring pendulum relative to due east. It seems unlikely, however, that this component of mooring motion is orders of magnitude larger than the azimuthal component.

Frequency-resolved structure functions should have a similar relative error because the mooring motion error is not random but rather strongly correlated with the flow itself. It should also be noted that the moorings were typically knocked synchronously, so while the instruments were located away from their rest depths, they were still located at approximately the same depths relative to one another. This means that the velocity differences still reflect largely horizontal rather than vertical shear, as intended. 

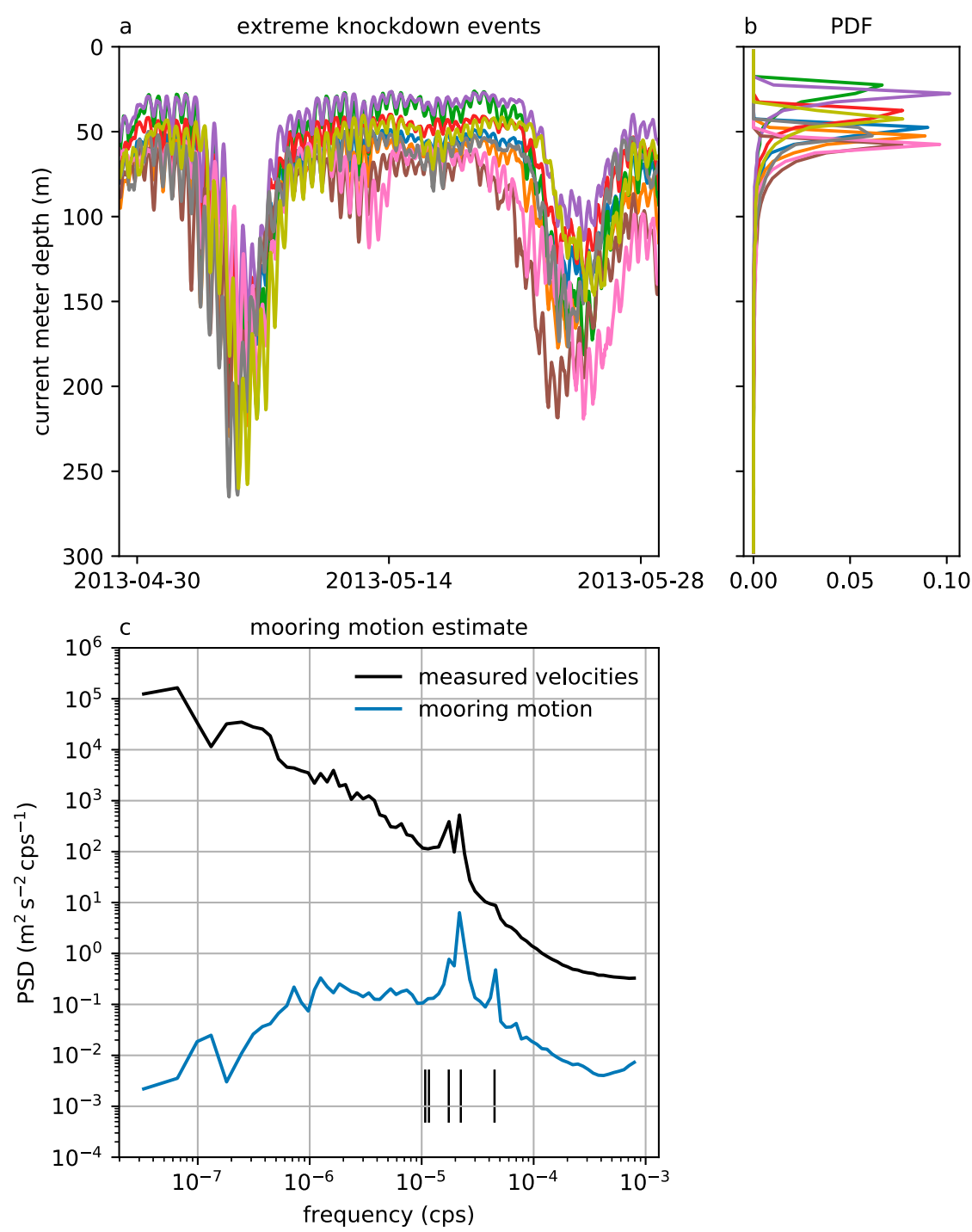

FIG. A1. Mooring knockdown and its impact on the frequency statistics. (a) Depths of the current meters with a nominal depth of $50 \mathrm{~m}$, as inferred from the pressure sensors, during a period of extreme knockdown. Each mooring is denoted with a different color. (b) Probability density functions of the instrument depths over the entire time series, indicating that the knockdown is more typically around $25 \mathrm{~m}$. (c) Kinetic energy frequency spectra estimated from the reported velocities and from the azimuthal mooring motion estimated using a stiff pendulum assumption.

\section{REFERENCES}

Babiano, A., C. Basdevant, and R. Sadourny, 1985: Structure functions and dispersion laws in two-dimensional turbulence. J. Atmos. Sci., 42, 941-949, https://doi.org/10.1175/ 1520-0469(1985)042<0941:SFADLI >2.0.CO;2.

Barkan, R., M. J. Molemaker, K. Srinivasan, J. C. McWilliams, and E. A. D'Asaro, 2019: The role of horizontal divergence in submesoscale frontogenesis. J. Phys. Oceanogr., 49, 15931618, https://doi.org/10.1175/JPO-D-18-0162.1.

Boccaletti, G., R. Ferrari, and B. Fox-Kemper, 2007: Mixed layer instabilities and restratication. J. Phys. Oceanogr., 37, 22282250, https://doi.org/10.1175/JPO3101.1.
Buckingham, C. E., and Coauthors, 2016: Seasonality of submesoscale flows in the ocean surface boundary layer. Geophys. Res. Lett., 43, 2118-2126, https://doi.org/10.1002/2016GL068009.

Bühler, O., J. Callies, and R. Ferrari, 2014: Wave-vortex decomposition of one-dimensional ship-track data. J. Fluid Mech., 756, 1007-1026, https://doi.org/10.1017/jfm.2014.488.

Callies, J., and R. Ferrari, 2013: Interpreting energy and tracer spectra of upper-ocean turbulence in the submesoscale range (1-200 km). J. Phys. Oceanogr., 43, 2456-2474, https://doi.org/ 10.1175/JPO-D-13-063.1.

,, , J. M. Klymak, and J. Gula, 2015: Seasonality in submesoscale turbulence. Nat. Commun., 6, 6862, https://doi.org/ 10.1038/ncomms7862. 
_ G. Flierl, R. Ferrari, and B. Fox-Kemper, 2016: The role of mixed-layer instabilities in submesoscale turbulence. J. Fluid Mech., 788, 5-41, https://doi.org/10.1017/jfm.2015.700.

Capet, X., J. C. McWilliams, M. J. Molemaker, and A. F. Shchepetkin, 2008: Mesoscale to submesoscale transition in the California current system. Part III: Energy balance and flux. J. Phys. Oceanogr., 38, 2256-2269, https://doi.org/10.1175/ 2008JPO3810.1.

Charney, J. G., 1971: Geostrophic turbulence. J. Atmos. Sci., 28, 1087-1095, https://doi.org/10.1175/1520-0469(1971)028<1087: $\mathrm{GT}>2.0 . \mathrm{CO} ; 2$.

Chelton, D. B., M. G. Schlax, R. M. Samelson, J. T. Farrar, M. J. Molemaker, J. C. McWilliams, and J. Gula, 2019: Prospects for future satellite estimation of small-scale variability of ocean surface velocity and vorticity. Prog. Oceanogr., 173, 256-350, https://doi.org/10.1016/j.pocean. 2018.10.012.

Chereskin, T. K., C. B. Rocha, S. T. Gille, D. Menemenlis, and M. Passaro, 2019: Characterizing the transition from balanced to unbalanced motions in the southern California current. J. Geophys. Res. Oceans, 124, 2088-2109, https://doi.org/ 10.1029/2018JC014583.

Choi, J., A. Bracco, R. Barkan, A. F. Shchepetkin, J. C. McWilliams, and J. M. Molemaker, 2017: Submesoscale dynamics in the northern Gulf of Mexico. Part III: Lagrangian implications. J. Phys. Oceanogr., 47, 2361-2376, https://doi.org/10.1175/JPOD-17-0036.1.

D'Asaro, E. A., and Coauthors, 2018: Ocean convergence and the dispersion of flotsam. Proc. Natl. Acad. Sci. USA, 115, 11621167, https://doi.org/10.1073/pnas.1718453115.

Erickson, Z. K., A. F. Thompson, J. Callies, X. Yu, A. Naveira Garabato, and P. Klein, 2020: The vertical structure of openocean submesoscale variability during a full seasonal cycle. J. Phys. Oceanogr., 50, 145-160, https://doi.org/10.1175/JPOD-19-0030.1.

Evans, D. G., and Coauthors, 2018: Annual cycle of turbulent dissipation estimated from seagliders. Geophys. Res. Lett., $\mathbf{4 5}$, 10 560-10 569, https://doi.org/10.1029/2018GL079966.

Ferrari, R., 2011: A frontal challenge for climate models. Science, 332, 316-317, https://doi.org/10.1126/science.1203632.

_ Reservoirs, sources, and sinks. Annu. Rev. Fluid Mech., 41, 253282, https://doi.org/10.1146/annurev.fluid.40.111406.102139.

Fox-Kemper, B., R. Ferrari, and R. W. Hallberg, 2008: Parameterization of mixed layer eddies. Part I: Theory and diagnosis. J. Phys. Oceanogr., 38, 1145-1165, https://doi.org/10.1175/2007JPO3792.1.

LaCasce, J. H., 2016: Estimating Eulerian energy spectra from drifters. Fluids, 1, 33, https://doi.org/10.3390/FLUIDS1040033.

Le Traon, P.-Y., P. Klein, B. L. Hua, and G. Dibarboure, 2008: Do altimeter wavenumber spectra agree with the interior or surface quasigeostrophic theory? J. Phys. Oceanogr., 38, 11371142, https://doi.org/10.1175/2007JPO3806.1.

Lindborg, E., 2015: A Helmholtz decomposition of structure functions and spectra calculated from aircraft data. J. Fluid Mech., 762, R4, https://doi.org/10.1017/jfm.2014.685.

Mahadevan, A., 2016: The impact of submesoscale physics on primary productivity of Plankton. Annu. Rev. Mar. Sci., 8, 161-184, https://doi.org/10.1146/annurev-marine-010814-015912.

McWilliams, J. C., 2016: Submesoscale currents in the ocean. Proc. Roy. Soc. London, A472, 20160117, https://doi.org/10.1098/ RSPA.2016.0117.

Mensa, J. A., Z. Garraffo, A. Griffa, T. M. Özgökmen, A. Haza, and M. Veneziani, 2013: Seasonality of the submesoscale dy- namics in the Gulf Stream region. Ocean Dyn., 63, 923-941, https://doi.org/10.1007/s10236-013-0633-1.

Molemaker, M. J., J. C. McWilliams, and X. Capet, 2010: Balanced and unbalanced routes to dissipation in an equilibrated Eady flow. J. Fluid Mech., 654, 35-63, https://doi.org/ 10.1017/S0022112009993272.

Müller, P., J. McWilliams, and J. Molemaker, 2005: Routes to dissipation in the ocean: The two-dimensional/three-dimensional turbulence conundrum. Marine Turbulence: Theories, Observations, and Models, H. Z. Baumert, J. Simpson, and J. Sündermann, Eds., Cambridge University Press, 397-405.

Omand, M. M., E. A. D'Asaro, C. M. Lee, M. J. Perry, N. Briggs, I. Cetinić, and A. Mahadevan, 2015: Eddy-driven subduction exports particulate organic carbon from the spring bloom. Science, 348, 222-225, https://doi.org/10.1126/science. 1260062.

Pearson, J., B. Fox-Kemper, R. Barkan, J. Choi, A. Bracco, and J. C. McWilliams, 2019: Impacts of convergence on structure functions from surface drifters in the Gulf of Mexico. J. Phys. Oceanogr., 49, 675-690, https://doi.org/10.1175/JPO-D-18-0029.1.

Pedlosky, J., 1987: Geophysical Fluid Dynamics. 2nd ed. Springer, $710 \mathrm{pp}$.

Qiu, B., S. Chen, P. Klein, J. Wang, H. Torres, L.-L. Fu, and D. Menemenlis, 2018: Seasonality in transition scale from balanced to unbalanced motions in the World Ocean. J. Phys. Oceanogr., 48, 591-605, https://doi.org/10.1175/JPO-D-17-0169.1.

Rocha, C. B., T. K. Chereskin, S. T. Gille, and D. Menemenlis, 2016: Mesoscale to submesoscale wavenumber spectra in Drake Passage. J. Phys. Oceanogr., 46, 601-620, https:// doi.org/10.1175/JPO-D-15-0087.1.

, G. L. Wagner, and W. R. Young, 2018: Stimulated generation: Extraction of energy from balanced ow by near-inertial waves. J. Fluid Mech., 847, 417-451, https://doi.org/10.1017/jfm.2018.308.

Sasaki, H., P. Klein, B. Qiu, and Y. Sasai, 2014: Impact of oceanicscale interactions on the seasonal modulation of ocean dynamics by the atmosphere. Nat. Commun., 5, 5636, https:// doi.org/10.1038/ncomms6636.

Savage, A. C., and Coauthors, 2017: Spectral decomposition of internal gravity wave sea surface height in global models. J. Geophys. Res. Oceans, 122, 7803-7821, https://doi.org/ 10.1002/2017JC013009.

Shcherbina, A. Y., E. A. D'Asaro, C. M. Lee, J. M. Klymak, M. J. Molemaker, and J. C. McWilliams, 2013: Statistics of vertical vorticity, divergence, and strain in a developed submesoscale turbulence field. Geophys. Res. Lett., 40, 4706-4711, https:// doi.org/10.1002/grl.50919.

Thomas, J., K. S. Smith, and O. Bühler, 2017: Near-inertial wave dispersion by geostrophic flows. J. Fluid Mech., 817, 406-438, https://doi.org/10.1017/jfm.2017.124.

Thomas, L. N., A. Tandon, and A. Mahadevan, 2008: Submesoscale processes and dynamics. Ocean Modeling in an Eddying Regime, Geophys. Monogr., Vol. 177, Amer. Geophys. Union, 17-38.

Thompson, A. F., A. Lazar, C. Buckingham, A. C. Naveira Garabato, G. M. Damerell, and K. J. Heywood, 2016: Openocean submesoscale motions: A full seasonal cycle of mixed layer instabilities from gliders. J. Phys. Oceanogr., 46, 12851307, https://doi.org/10.1175/JPO-D-15-0170.1.

Torres, H. S., P. Klein, D. Menemenlis, B. Qiu, Z. Su, J. Wang, S. Chen, and L.-L. Fu, 2018: Partitioning ocean motions into balanced motions and internal gravity waves: A modeling study in anticipation of future space missions. J. Geophys. Res. Oceans, 123, 8084-8105, https://doi.org/ 10.1029/2018JC014438. 
Wang, D.-P., C. N. Flagg, K. Donohue, and H. T. Rossby, 2010: Wavenumber spectrum in the Gulf Stream from shipboard ADCP observations and comparison with altimetry measurements. J. Phys. Oceanogr., 40, 840-844, https://doi.org/ 10.1175/2009JPO4330.1.

Wunsch, C., 2009: The oceanic variability spectrum and transport trends. Atmos.-Ocean, 47, 281-291, https://doi.org/10.3137/ OC310.2009.

_- and D. Stammer, 1995: The global frequencywavenumber spectrum of oceanic variability estimated from TOPEX/POSEIDON altimetric measurements. J. Geophys. Res., 100, 24 895-24 910, https://doi.org/10.1029/ 95JC01783.
Xie, J.-H., and J. Vanneste, 2015: A generalised-Lagrangian-mean model of the interactions between near-inertial waves and mean flow. J. Fluid Mech., 774, 143-169, https://doi.org/10.1017/jfm.2015.251.

Yaglom, A. M., 1962: An Introduction to the Theory of Stationary Random Functions. Prentice-Hall Inc., 235 pp.

Young, W. R., and M. Ben Jelloul, 1997: Propagation of nearinertial oscillations through a geostrophic flow. J. Mar. Res., 55, 735-766, https://doi.org/10.1357/0022240973224283.

Yu, X., A. C. Naveira Garabato, A. P. Martin, C. E. Buckingham, L. Brannigan, and Z. Su, 2019: An annual cycle of submesoscale vertical flow and restratification in the upper ocean. J. Phys. Oceanogr., 49, 1439-1461, https://doi.org/10.1175/ JPO-D-18-0253.1. 